\title{
Dressed asymptotic states and QED infrared physics
}

\author{
Hideo Furugori (古郡 秀雄) $\oplus^{1, *}$ and Shin'ichi Nojiri ${ }^{1,2, \dagger}$ \\ ${ }^{1}$ Department of Physics, Nagoya University, Nagoya 464-8602, Japan \\ ${ }^{2}$ Kobayashi-Maskawa Institute for the Origin of Particles and the Universe, \\ Nagoya University, Nagoya 464-8602, Japan
}

(Received 8 July 2020; revised 15 September 2021; accepted 24 October 2021; published 3 December 2021)

\begin{abstract}
The dressed-state formalisms, which incorporate interactions of soft particles into an asymptotic state, are known as the prescriptions expected to solve the problem of infrared (IR) divergence in the quantum field theory (QFT). A particularly famous example is the dressed-state formalism proposed by Kulish and Faddeev in quantum electrodynamics (QED). As pointed out by Hirai and Sugishita, however, this formalism has problems in gauge invariance and the IR divergence. These problems are mainly caused by the existence of ghosts or unphysical photon modes. Therefore, we start by studying the asymptotic states in the Coulomb gauge, which excludes ghosts and/or unphysical photon modes. In this paper, we propose a formalism to construct the asymptotic states directly from the interaction of the theory by setting a sufficiently large timescale $T$. In this dressed-state formalism, we define the asymptotic interaction remaining at $|t|>T$ in terms of some fixed order of $1 / T$, and we are performing all calculations according to that order. We study the asymptotic states in QED specifically, but we can formally apply the dressedstate formalism proposed in this paper to any perturbative QFT. We show that, at least in QED, we can construct a divergence-free and unitary $S$-matrix using dressed states proposed in this paper. Furthermore, we discuss the transition rate to show that we can predict experimental results. We also show that the $\mathrm{U}(1)$ gauge symmetry of the $S$-matrix leads to the QED large gauge symmetry, and the deviation of the expectation values of the vector potential between initial and final spacelike hypersurfaces emerges as a QED memory effect. The dressed-state formalism in this paper may give a unified and new insight into IR physics, including asymptotic symmetries, memory effects, and unitarity of the state evolution.
\end{abstract}

DOI: 10.1103/PhysRevD.104.125004

\section{INTRODUCTION}

The problem of the infrared (IR) divergence in the transition amplitudes is well known in the gauge theories in four dimensions. In quantum electrodynamics (QED), for example, this is a problem where loop corrections coming from low-energy photons (i.e., soft photons) give an infinite phase to the transition amplitudes and make the transition probabilities approach zero due to the IR divergences. This problem has been solved by using the BlochNordsieck (BN) formalism [1], where we assume that the physical final state is a superposition of states with any number of soft photons, because we cannot distinguish between the final state and states adding an arbitrary number of soft photons to the final state. In the BN formalism, the soft-photon theorem, which connects amplitudes emitting

\footnotetext{
*furugori.hideo@a.mbox.nagoya-u.ac.jp

†nojiri@gravity.phys.nagoya-u.ac.jp
}

Published by the American Physical Society under the terms of the Creative Commons Attribution 4.0 International license. Further distribution of this work must maintain attribution to the author(s) and the published article's title, journal citation, and DOI. Funded by SCOAP ${ }^{3}$. soft photons with the original amplitude, is essential to recovering the predictability of the theory $[2,3]$.

Interestingly, it has been discovered that this soft-photon theorem is related to the asymptotic symmetry of the theory [4-7]. Adding to the memory effect, the relations between soft theorems and asymptotic symmetries and memory effects have recently become known as the "IR triangle," which is now actively investigated (for example, see Ref. [8]). In the study, the asymptotic symmetry of QED called large gauge symmetry implies the existence of an infinite number of large charges with functional degrees of freedom. By choosing the function of the large charge to be a specific one and using the Ward-Takahashi identity, we can derive the soft-photon theorem. A similar relation between the Bondi-van der Burg-Metzner-Sachs (BMS) asymptotic symmetry $[9,10]$ and linearized gravity theory has been found [11]. These studies lead to the consideration that asymptotic symmetries shed new light on the information-loss problem of the black hole (BH) [12]. Their idea is that an infinite number of soft charges could also exist on the $\mathrm{BH}$, which generates soft photons and soft gravitons (and so on) in quantum theory, and these soft particles may protect the unitarity of the time evolution from the 
formation to the evaporation of the $\mathrm{BH}$. In other words, the existence of the soft particles which save the IR divergence problem for transition processes in flat spacetime may play an important role even in curved spacetime. We should note, however, that in the $\mathrm{BN}$ formalism, we cannot remove the IR divergence in the $S$-matrix, although there is no divergence in the transition probability. In other words, the unitarity of the quantum process is not guaranteed. Therefore, it is appropriate that we use the dressed-state formalisms [13-21], by which we expect to construct an IR-divergence-free $S$-matrix so that we can check the unitarity.

In the dressed-state formalisms, we do not take asymptotic states as free-particle states, but dressed states with an infinite number of soft particles. A particularly famous example is the dressed-state formalism by Kulish and Faddeev (KF) in QED [18]. Recalling that the soft theorem is derived from one of the large charges in the context of asymptotic symmetries, we can see that the theorem is not equivalent to the asymptotic symmetry but is included by the asymptotic symmetry. Furthermore, there have been several studies showing that the dressed states are eigenstates of the large charges and naturally arise by guaranteeing the conservation of the large charges [22,23]. Hence, we can expect that dressed-state formalisms may play an important role not only in the unitarity of the state evolution but also in the understanding of asymptotic symmetries. On the other hand, as pointed out by Hirai and Sugishita [21], the dressed states given by Kulish and Faddeev have some problems in the gauge invariance and the IR divergence. In other words, we should construct a more suitable dressedstate formalism than the KF formalism.

Motivated by the above situation, we propose a dressedstate formalism and study the asymptotic states, $S$-matrix, transition rate, gauge symmetry, and memory effect in QED. The contents of this paper are as follows: In the next section, we briefly review the scattering problem in quantum field theory (QFT). Next, we review the KF formalism and point out some problems. Especially, we mention an important problem of the condition for the gauge invariance pointed out by Hirai and Sugishita [21]. In Sec. III, We propose a dressedstate formalism to solve the problems and give the theoryindependent definition of asymptotic states and the $S$-matrix. After that, in Sec. IV, we apply the dressed-state formalism to QED as a specific example, and we explicitly show that there is no divergence in the $S$-matrix proposed in this paper and give a physical transition rate. We also discuss the gauge symmetry and memory effect. The last section is devoted to the summary and prospects.

\section{REVIEW ON KULISH-FADDEEV DRESSED-STATE FORMALISM AND THE PROBLEMS}

Although there are many papers on the dressed-state formalism, the terminology and formulations used in the papers are not so unified. In this section, we review the paper by Kulish and Faddeev [18] in some detail and clarify the terminology which we use to avoid confusion.

\section{A. Fock-based $S$-matrix}

First, we recall the standard scattering problem in QFT. In the scattering problem, we predict the transition probability from an "in" state $\left|\Psi_{\alpha}^{+}\right\rangle$in the sufficiently far past ${ }^{1}$ to an "out" state $\left|\Psi_{\beta}^{-}\right\rangle$in the sufficiently far future caused by the scatterings. ${ }^{2}$ Here, $\left|\Psi_{\alpha}^{+}\right\rangle$and $\left|\Psi_{\beta}^{-}\right\rangle$are eigenstates of the Hamiltonian of the system with the energies $E_{\alpha}$ and $E_{\beta}$, respectively. ${ }^{3}$ We may decompose the Hamiltonian $H$ into the free Hamiltonian $H_{0}$ and express the interaction $V$ as $H=H_{0}+V$. It is difficult to follow the time evolution of states moment by moment in general if $V$ exists. By assuming that the interaction is sufficiently weak in the far past and the far future, we can use the particle picture and calculate the $S$-matrix, which is defined by

$$
S_{\beta \alpha}:=\left\langle\Psi_{\beta}^{-} \mid \Psi_{\alpha}^{+}\right\rangle
$$

In the particle picture, the "in" and "out" states asymptotically correspond to the free-particle state $\left|\Phi_{\alpha}\right\rangle$ in a free theory. By using the interaction picture, ${ }^{4}$ this correspondence can be seen as

$$
\lim _{t \rightarrow-\infty}\left|\Psi_{\alpha}^{+}(t)\right\rangle_{\mathrm{I}}:=\lim _{t \rightarrow-\infty} \Omega(t)\left|\Psi_{\alpha}^{+}\right\rangle \simeq\left|\Phi_{\alpha}\right\rangle .
$$

Here, $\Omega(t)$ is a unitary operator connecting the fields in the Heisenberg picture and the fields in the interaction picture, so that

$$
\mathcal{O}(\vec{x}, t)=\Omega^{\dagger}(\vec{x}, t) \mathcal{O}^{\mathrm{I}}(\vec{x}, t) \Omega(\vec{x}, t) .
$$

We can also express the asymptotic "out" state similarly, and the $S$-matrix in the particle picture is given by

$$
\begin{aligned}
\left\langle\Psi_{\beta}^{-} \mid \Psi_{\alpha}^{+}\right\rangle & \equiv\left\langle\Psi_{\beta}^{-}\left|\lim _{t^{\prime} \rightarrow \infty} \Omega^{\dagger}\left(t^{\prime}\right) \Omega\left(t^{\prime}\right) \lim _{t \rightarrow-\infty} \Omega^{\dagger}(t) \Omega(t)\right| \Psi_{\alpha}^{+}\right\rangle \\
& \stackrel{!}{\simeq}\left\langle\Phi_{\beta}\left|\mathcal{S}_{\mathrm{D}}\right| \Phi_{\alpha}\right\rangle=: S_{\beta \alpha}^{\mathrm{D}} .
\end{aligned}
$$

Here, $\mathcal{S}_{\mathrm{D}}$ is the Dyson $S$-operator defined by

\footnotetext{
${ }^{1} \mathrm{We}$ set the reference time to the time origin $t=0$.

${ }^{2}$ Here we use the Heisenberg picture.

${ }^{3}$ We should note, however, that if these states are completely the eigenstates of the Hamiltonian, no scattering occurs. Rigorously, we therefore need to consider the transition from a wave packet $\mid$ in $\rangle$ to another wave packet $\mid$ out $\rangle$, which is defined by

$$
\left.\mid \text { in }\rangle:=\int d \alpha g_{\text {in }}(\alpha)\left|\Psi_{\alpha}^{+}\right\rangle, \quad \mid \text { out }\right\rangle:=\int d \beta g_{\text {out }}(\beta)\left|\Psi_{\beta}^{-}\right\rangle .
$$
}

Here, $g_{\text {in }}(\alpha)$ and $g_{\text {out }}(\beta)$ are amplitudes that do not vanish and smoothly varying over some finite range $\Delta E$ of energies. In this paper, however, we do not analyze by using the packets.

${ }^{4}$ We use the suffix "I" to express the interaction picture. 


$$
\mathcal{S}_{\mathrm{D}}:=\lim _{\substack{t^{\prime} \rightarrow \infty \\ t \rightarrow-\infty}} \Omega\left(t^{\prime}\right) \Omega^{\dagger}(t)=\mathcal{T} \exp \left[-i \int_{-\infty}^{\infty} d \tau V^{\mathrm{I}}(\tau)\right]
$$

The notation of $\mathcal{T}$ expresses the time-ordered product. The symbol "!" in Eq. (2.4) denotes caution that we should take care of the validity. Note that the last expression is derived by taking the limit $|t| \rightarrow \infty$ before the unitary operator $\Omega(t)$ acts on the states. That is the starting point of our method, which we will see later. We also define $S_{\beta \alpha}^{\mathrm{D}}$ as the standard Fock-based $S$-matrix calculated by using Dyson $S$-operator. Equation (2.4) is the standard expression for the $S$-matrix in the particle picture. Since free-particle states are in the Fock space $\mathscr{H}_{\mathrm{F}}, \mathcal{S}_{\mathrm{D}}$ is the map from $\mathscr{H}_{\mathrm{F}}$ to $\mathscr{H}_{\mathrm{F}}$. The $S$-matrix $S_{\beta \alpha}^{\mathrm{D}}$, sandwiched in the Fock states, is not well defined in QED due to the existence of the IR divergence. The IR-divergence problem in QED is that quantum corrections from soft photons attach an infinitely large phase factor and an infinitely small coefficient to the $S$-matrix as in Eq. (4.17).

\section{B. Effects of the asymptotic interaction and the Kulish-Faddeev $S$-operator}

The problem of an infinite phase appearing in the $S$-matrix was also known in the Coulomb scattering in nonrelativistic quantum mechanics. One way to solve this problem is known as the Dollard formalism [24], which redefines the $S$-matrix incorporating the non-negligible influence of the Coulomb interaction, even at sufficiently far distances, as a cause of the problem. By applying this formalism to relativistic field theory, Kulish and Faddeev have shown that the asymptotic state of QED is not a free-particle state but a dressed state clinging by countless photons and have used it to discuss the IR divergent-free $S$-matrix. In other words, the dressedstate formalism is a way to regard infrared divergence as a breakdown of the particle picture.

Now, we will see the dressed-state formalism by Kulish and Faddeev. The QED Lagrangian they used is the Lorentz covariant form as follows:

$$
\mathcal{L}=-\frac{1}{4} F_{\mu \nu} F^{\mu \nu}-\frac{1}{2 \alpha}\left(\partial^{\mu} A_{\mu}\right)^{2}-\bar{\Psi}\left(\gamma^{\mu} \partial_{\mu}+m\right) \Psi-i e \bar{\Psi} \gamma^{\mu} \Psi A_{\mu}+\mathcal{L}_{\mathrm{c}}
$$

Here, $\mathcal{L}_{\mathrm{c}}$ is a counterterm for the renormalization. Taking the Feynman gauge $(\alpha=1)$ and moving to the canonical formalism, we can find that the vector field $\tilde{a}_{\mu}(x):=\Omega^{\dagger}(t) A_{\mu}(x) \Omega(t)$ and spinor field $\psi(x):=\Omega^{\dagger}(t) \Psi(x) \Omega(t)$ with charge $e$ in the interaction picture are given by

$$
\begin{aligned}
& \tilde{a}_{\mu}(x)=\int \frac{d^{3} k}{(2 \pi)^{3 / 2} \sqrt{2 \omega}} \sum_{\tilde{h}}\left[\epsilon_{\mu}(\vec{k}, \tilde{h}) \mathrm{e}^{i k \cdot x} a(\vec{k}, \tilde{h})+\epsilon_{\mu}^{*}(\vec{k}, \tilde{h}) \mathrm{e}^{-i k \cdot x} a^{\dagger}(\vec{k}, \tilde{h})\right], \quad k^{0}=|\vec{k}|=: \omega, \\
& \psi(x)=\int \frac{d^{3} p}{(2 \pi)^{3 / 2}} \sum_{\sigma}\left[u_{\sigma}(\vec{p}) \mathrm{e}^{i p \cdot x} b_{\sigma}(\vec{p})+v_{\sigma}(\vec{p}) \mathrm{e}^{-i p \cdot x} d_{\sigma}^{\dagger}(\vec{p})\right], \quad p^{0}=\sqrt{m^{2}+|\vec{p}|^{2}}=: E_{p} .
\end{aligned}
$$

Here, $h=\{+,-\}$ denotes transverse mode with helicity \pm 1 , and $I=\{\mathrm{S}, \mathrm{L}\}$ denotes the scalar mode and longitudinal mode, respectively, and we define $\tilde{h}=\{h, I\}$. Hereafter, we use the tilde (c) to remind the reader that unphysical modes $I$ are included. In Eq. (2.7), $a(\vec{k}, \tilde{h})$ is the annihilation operator of the $\tilde{h}$-mode photon with momentum $\vec{k}$, and $\epsilon_{\mu}(\vec{k}, \tilde{h})$ is the polarization vector of the photon. On the other hand, $b_{\sigma}(\vec{p})$ and $d_{\sigma}(\vec{p})$ are the annihilation operators of the electron and the positron with spin $\sigma$ and momentum $\vec{p}$, respectively. We put some notes in Appendix A for the properties of the polarization vector $\epsilon_{\mu}(\vec{k}, \tilde{h})$ and coefficient functions of the spinor field $u_{\sigma}(\vec{p}), v_{\sigma}(\vec{p})$. In this theory, QED interacion is given by ${ }^{5}$

$$
\tilde{V}^{\mathrm{I}}(t)=i e \int d^{3} x \tilde{a}_{\mu}(x) \bar{\psi}(x) \gamma^{\mu} \psi(x) .
$$

Kulish and Faddeev have considered the asymptotic interaction $\tilde{V}_{\text {as }}^{\mathrm{I}}$, which we should take into account even in the far past or future. By writing down the QED interaction explicitly by using the field expressions in Eqs. (2.7) and (2.8), we get $^{6}$

$$
\begin{aligned}
\tilde{V}^{\mathrm{I}}(t)= & i e \int d^{3} x \sum_{\tilde{h}, \sigma, \sigma^{\prime}} \int \frac{d^{3} k d^{3} p d^{3} q}{(2 \pi)^{9 / 2} \sqrt{2 \omega}}\left[a_{\mu}(\vec{k}, \tilde{h}) \mathrm{e}^{i k \cdot x}\right. \\
& \times\left\{\mathrm{e}^{i(p+q) \cdot x} \bar{v}_{\sigma}(\vec{p}) \gamma^{\mu} u_{\sigma^{\prime}}(\vec{q}) d_{\sigma}(\vec{p}) b_{\sigma^{\prime}}(\vec{q})+\mathrm{e}^{-i(p+q) \cdot x} \bar{u}_{\sigma}(\vec{p}) \gamma^{\mu} v_{\sigma^{\prime}}(\vec{q}) b_{\sigma}^{\dagger}(\vec{p}) d_{\sigma^{\prime}}^{\dagger}(\vec{q})\right. \\
& \left.\left.+\mathrm{e}^{i(p-q) \cdot x} \bar{v}_{\sigma}(\vec{p}) \gamma^{\mu} v_{\sigma^{\prime}}(\vec{q}) d_{\sigma}^{\dagger}(\vec{p}) d_{\sigma^{\prime}}(\vec{q})+\mathrm{e}^{-i(p-q) \cdot x} \bar{u}_{\sigma}(\vec{p}) \gamma^{\mu} u_{\sigma^{\prime}}(\vec{q}) b_{\sigma}^{\dagger}(\vec{p}) b_{\sigma^{\prime}}(\vec{q})\right\}+ \text { (H.c.) }\right) .
\end{aligned}
$$

\footnotetext{
${ }^{5}$ We always use normal-ordered interaction.

${ }^{6}$ We also use the notation $a_{\mu}(k, \tilde{h}):=\epsilon_{\mu}(k, \tilde{h}) a(k, \tilde{h})$.
} 
By integrating the above expression over $\vec{x}$, the delta functions connecting the momenta appear. Further integrating over the momentum $\vec{q}$, we find that, in the second line of Eq. (2.10), the coefficients of $t$ in the exponents are given by $\omega \pm\left(E_{p}+E_{p \pm k}\right)$. We can neglect the second line in Eq. (2.10), since the coefficients are always positive and violently oscillate in the far past or future. On the other hand, we cannot neglect the corresponding coefficients in the third line in Eq. (2.10), since the coefficients of $t$ in the exponents are given by $\omega \pm\left(E_{p}-E_{p \pm k}\right)$, and these terms go to zero in the $\omega=0$ regions. Kulish and Faddeev have defined the asymptotic interaction as a form where we set $\omega=0$-i.e.,

$$
\begin{gathered}
\tilde{V}_{\mathrm{as}}^{\mathrm{I}}(t):=e \sum_{\tilde{h}} \int \frac{d^{3} k}{(2 \pi)^{3 / 2} \sqrt{2 \omega}} \int d^{3} p \hat{\rho}(\vec{p}) v_{p}^{\mu}\left[a_{\mu}(\vec{k}, \tilde{h}) \mathrm{e}^{i k \cdot v_{p} t}+(\text { H.c. })\right], \\
v_{p}^{\mu}:=\frac{p^{\mu}}{E_{p}}, \quad E_{p}:=\sqrt{m^{2}+|\vec{p}|^{2}}, \quad \hat{\rho}(\vec{p}):=\sum_{\sigma}\left[b_{\sigma}^{\dagger}(\vec{p}) b_{\sigma}(\vec{p})-d_{\sigma}^{\dagger}(\vec{p}) d_{\sigma}(\vec{p})\right]
\end{gathered}
$$

is the asymptotic interaction of QED which we should consider. We note that the integration region of the photon momentum is unbounded. ${ }^{7}$ We can rewrite Eq. (2.11) as

$$
\tilde{V}_{\mathrm{as}}^{\mathrm{I}}(t)=\int d^{3} x \tilde{a}_{\mu}(x) j_{\mathrm{cl}}^{\mu}(x), \quad j_{\mathrm{cl}}^{\mu}(x)=e \int d^{3} p v_{p}^{\mu} \hat{\rho}(\vec{p}) \delta^{3}\left(\vec{x}-\overrightarrow{v_{p}} t\right)
$$

and now we can easily find that photons couple with individual moving charged particles in the far past or far future.

Following the Dollard formalism, Kulish and Faddeev have investigated the asymptotic states by considering the asymptotic time evolution with asymptotic Hamiltonian $H_{\text {as }}=H_{0}+\tilde{V}_{\text {as }}$ in the Schrödinger picture. ${ }^{8}$ Now, the operator $U_{\text {as }}(t)$, which describes the time development of the asymptotic states, satisfies

$$
i \frac{d U_{\mathrm{as}}(t)}{d t}=H_{\mathrm{as}}^{\mathrm{S}}(t) U_{\mathrm{as}}(t)
$$

which can be solved by assuming $U_{\text {as }}(t)=\mathrm{e}^{-i H_{0}^{\mathrm{S}}} \tilde{Z}(t)$. Then, $\tilde{Z}(t)$ satisfies the following equation:

$$
i \frac{d \tilde{Z}(t)}{d t}=\tilde{V}_{\mathrm{as}}^{\mathrm{I}}(t) \tilde{Z}(t)
$$

The solution is given by

$$
\tilde{Z}(t)=\mathcal{T} \exp \left[-i \int_{0}^{t} d \tau \tilde{V}_{\mathrm{as}}^{\mathrm{I}}(\tau)\right]
$$

\footnotetext{
${ }^{7}$ This results in the ultraviolet (UV) divergence coming from the high-momentum region. Kulish and Faddeev have assumed that the divergence could be removed by the renormalization of mass. However, there is room for discussion to deal with this UV divergence. For example, there is a study regarding the detection limit of photons as the upper cutoff of the momentum value [25]. Or, as we will propose later, we may regard the inverse of the timescale of the experiment as the upper cutoff.

${ }^{8}$ We use the suffix " $S$ " to express the Schrödinger picture.
}

By using the expressions in Eqs. (2.11) and (2.12), Eq. (2.16) can be explicitly rewritten as

$$
\begin{aligned}
\tilde{Z}(t)= & \exp \left[-i \int_{0}^{t} d \tau \tilde{V}_{\text {as }}^{\mathrm{I}}(\tau)\right. \\
& \left.-\frac{1}{2} \int_{0}^{t} d \tau \int_{0}^{\tau} d \tau^{\prime}\left[\tilde{V}_{\text {as }}^{\mathrm{I}}(\tau), \tilde{V}_{\text {as }}^{\mathrm{I}}\left(\tau^{\prime}\right)\right]\right]=: \mathrm{e}^{\hat{\hat{R}}(t)} \mathrm{e}^{i \hat{\theta}(t)} .
\end{aligned}
$$

The first term in the exponent is nowadays often called the dress operator $\hat{\tilde{R}}(t)$ :

$$
\hat{\tilde{R}}(t):=\int d^{3} k \int d^{3} p \sum_{\tilde{h}}\left[\hat{f}(p, k, \tilde{h} ; t) a^{\dagger}(\vec{k}, \tilde{h})-(\text { H.c. })\right],
$$

$\hat{\tilde{f}}(p, k, \tilde{h} ; t):=\frac{e \hat{\rho}(\vec{p})}{(2 \pi)^{3 / 2} \sqrt{2 \omega}} \frac{p \cdot \epsilon^{*}(k, \tilde{h})}{k \cdot p} \mathrm{e}^{-i k \cdot v_{p} t}$.

Here, Kulish and Faddeev have removed a term $\hat{\tilde{R}}(t=0)$ coming from the lower limit of the integration in Eq. (2.17), due to the consideration that conditions at $t=0$ should not affect asymptotic states. For later use, we define

$$
\begin{aligned}
D_{\hat{\hat{f}}(t)}:= & \exp \left[\int d ^ { 3 } k \int d ^ { 3 } p \sum _ { \tilde { h } } \left[\hat{\tilde{f}}(p, k, \tilde{h} ; t) a^{\dagger}(\vec{k}, \tilde{h})\right.\right. \\
& -(\text { H.c. })]] .
\end{aligned}
$$

This operator is just a displacement operator for defining coherent states. We put some notes in Appendix B for the properties of coherent states in nonrelativistic quantum mechanics. The second term in the exponent of Eq. (2.17) is called the phase operator $i \hat{\theta}(t)$, which gives an infinitely 
large phase, and the explicit form is expressed by

$$
i \hat{\theta}(t):=i \frac{\mathrm{e}^{2}}{8 \pi} \int d^{3} p \int d^{3} q \hat{\rho}(\vec{p}) \hat{\rho}(\vec{q}) \frac{p \cdot q}{\sqrt{(p \cdot q)-m^{4}}} \int_{0}^{t} \frac{d \tau}{\tau} .
$$

Now, we know the explicit form of $U_{\text {as }}$, and Kulish and Faddeev have defined the $S$-operator as follows:

$$
\begin{aligned}
\mathcal{S}_{\mathrm{KF}} & :=\lim _{t^{\prime} \rightarrow \infty, t \rightarrow-\infty} \mathcal{S}_{\mathrm{KF}}^{\mathrm{as}}\left(t^{\prime}, t\right), \\
\mathcal{S}_{\mathrm{KF}}^{\mathrm{as}}\left(t^{\prime}, t\right) & :=Z^{\dagger}\left(t^{\prime}\right) \mathcal{S}\left(t^{\prime}, t\right) Z(t), \\
\mathcal{S}\left(t^{\prime}, t\right) & :=\mathcal{T} \exp \left[-i \int_{t}^{t^{\prime}} d \tau V^{\mathrm{I}}(\tau)\right] .
\end{aligned}
$$

Since the dress operator $\hat{\tilde{R}}(t)$ goes to zero when we take the limit $t \rightarrow \pm \infty$ because we have the relation

$$
\lim _{t \rightarrow \pm \infty} \frac{1}{v_{p} \cdot k} \mathrm{e}^{i k \cdot v_{p} t}= \pm i \pi \delta\left(k \cdot v_{p}\right)=0,
$$

we get

$$
\mathcal{S}_{\mathrm{KF}} \stackrel{!}{=} \mathrm{e}^{-i \hat{\theta}(\infty)} \mathcal{S}_{\mathrm{D}} \mathrm{e}^{i \hat{\theta}(-\infty)}
$$

if we can take the limit $|t| \rightarrow \infty$ in advance.

\section{Kulish-Faddeev asymptotic states and their $S$-matrix}

Kulish and Faddeev have discussed the property of the Hilbert space $\mathscr{H}_{\text {as }}$ where their asymptotic states exist by considering that $\mathcal{S}_{\mathrm{KF}}^{\text {as }}$ is the map from $\mathscr{H}_{\text {as }}$ to $\mathscr{H}_{\text {as }}$. Assuming that $\mathcal{S}\left(t^{\prime}, t\right)$ is the map from $\mathscr{H}_{\mathrm{F}}$ to $\mathscr{H}_{\mathrm{F}}$, we can regard $\mathscr{H}_{\text {as }}$ as

$$
\mathscr{H}_{\mathrm{as}}=\tilde{Z}^{\dagger}(t) \mathscr{H}_{\mathrm{F}} \equiv D_{\hat{\tilde{f}}(t)}^{\dagger} \mathscr{H}_{\mathrm{F}}=D_{-\hat{f}(t)} \mathscr{H}_{\mathrm{F}}=\mathrm{e}^{-\hat{\hat{R}}(t)} \mathscr{H}_{\mathrm{F}},
$$

because $^{9} \tilde{Z}(t) \tilde{Z}^{\dagger}(t)=1$. Since the dress operator and the phase operator do not mix the charged particles and the photons, it is useful to decompose the Hilbert space into the Hilbert space of charged particles and that of photons like $\mathscr{H}_{\mathrm{F}}=\mathscr{H}_{\psi} \otimes \mathscr{H}_{\gamma}$. Then, we can express the vacuum state as a direct product of the vacuum state of the charged particle and the vacuum state of the photon, such that $|0\rangle=:|0 ; \psi\rangle \otimes|0 ; \gamma\rangle$. To see the properties of the asymptotic states, we concretely consider the situation where the asymptotic state in the particle picture is given by

\footnotetext{
${ }^{9}$ Now, we consider the case that $\tilde{Z}(t)$ is not a well-defined operator on $\mathscr{H}_{\mathrm{F}}$. Indeed, as we will see soon, $\tilde{Z}(t)$ is not a welldefined operator on $\mathscr{H}_{\mathrm{F}}$ for states with charged particles.
}

$$
\begin{aligned}
\left|\Phi_{\alpha}\right\rangle= & \left|\psi_{\alpha}\right\rangle \otimes|0 ; \gamma\rangle:=b_{\sigma_{1}}^{\dagger}\left(\vec{p}_{1}\right) \cdots b_{\sigma_{N}}^{\dagger}\left(\vec{p}_{N}\right) d_{s_{1}}^{\dagger}\left(\vec{q}_{1}\right) \\
& \cdots d_{s_{M}}^{\dagger}\left(\vec{q}_{M}\right)|0 ; \psi\rangle \otimes|0 ; \gamma\rangle .
\end{aligned}
$$

Now, we find

$$
D_{\hat{\tilde{f}}(t)}^{\dagger}\left|\Phi_{\alpha}\right\rangle=\left|\psi_{\alpha}\right\rangle \otimes\left|-\tilde{f}_{\alpha}(t)\right\rangle, \quad\left|-\tilde{f}_{\alpha}(t)\right\rangle:=D_{\tilde{f}_{\alpha}}^{\dagger}(t)|0 ; \gamma\rangle,
$$

$$
\begin{aligned}
D_{\tilde{f}_{\alpha}(t)}^{\dagger} & =D_{-\tilde{f}_{\alpha}(t)} \\
& :=\exp \left[-\sum_{\tilde{h}} \int d^{3} k\left[f_{\alpha}(k, \tilde{h} ; t) a^{\dagger}(\vec{k}, \tilde{h})-(\text { H.c. })\right]\right],
\end{aligned}
$$

$$
\begin{aligned}
\tilde{f}_{\alpha}(k, \tilde{h} ; t) & :=\sum_{n \in \alpha} \frac{e_{n}}{(2 \pi)^{3 / 2} \sqrt{2 \omega}} \frac{p_{n} \cdot \epsilon^{*}(k, \tilde{h})}{k \cdot p_{n}} \mathrm{e}^{-i k \cdot v_{n} t}, \\
v_{n}^{\mu} & :=p_{n}^{\mu} / E_{n}, \quad E_{n}:=\sqrt{m^{2}+\left|\vec{p}_{n}\right|^{2}} .
\end{aligned}
$$

Here, $n$ is the label of the charged particle in the asymptotic state, and $e_{n}$ is the electric charge of the $n$th particle. We note that the asymptotic photon state $\left|-\tilde{f}_{\alpha}(t)\right\rangle$ is just a coherent state. We use the expression Eq. (2.29) when we add the species of charged particles by changing mass $m \rightarrow m_{n}$. We should note, however, that we cannot apply the properties of coherent states straightforwardly when we add the hard photon in the states, because their asymptotic states contain photons with arbitrary momenta. As in the quantum-mechanical case, we can deform $D_{\tilde{f}_{\alpha}(t)}^{\dagger}$ to the normal-ordered form:

$$
\begin{aligned}
D_{\tilde{f}_{\alpha}(t)}^{\dagger}= & \exp \left[-\frac{1}{2} \sum_{\tilde{h}} \int d^{3} k\left|\tilde{f}_{\alpha}(k, \tilde{h} ; t)\right|^{2}\right] \\
& \times \exp \left[\sum_{\tilde{h}} \int d^{3} k \tilde{f}_{\alpha}(k, \tilde{h} ; t) a^{\dagger}(k, \tilde{h})\right] \\
& \times \exp \left[-\sum_{\tilde{h}} \int d^{3} k \tilde{f}_{\alpha}^{*}(k, \tilde{h} ; t) a(k, \tilde{h})\right] .
\end{aligned}
$$

If there exist charged particles in the state of the particle picture, $D_{\tilde{f}_{\alpha}(t)}^{\dagger}$ is not a well-defined operator on the Fock space, because we find

$$
\sum_{\tilde{h}} \int d^{3} k\left|\tilde{f}_{\alpha}(k, \tilde{h} ; t)\right|^{2}=\infty .
$$

Hence, $\mathscr{H}_{\text {as }} \neq \mathscr{H}_{\mathrm{F}}$ in general. ${ }^{10}$ Recalling the nonrelativistic case, we can understand that the asymptotic states of

\footnotetext{
${ }^{10}$ If there is no charged particle in the state of the particle picture, the asymptotic state exists in the Fock space, since $\tilde{f}_{\alpha}=0$. The Hilbert space $\mathscr{H}_{\text {as }}$, called the von Neumann space, is larger than the Fock space.
} 
QED are the dressed state with an infinite number of photons, because the lhs of Eq. (2.31) represents the averaged number of photons.

Kulish and Faddeev have chosen the state $\left.\left|\Psi_{\alpha}^{\mathrm{KF}}\right\rangle\right\rangle$ as a physically suitable asymptotic state which belongs to $\mathscr{H}_{\text {as }}^{\text {phys }} \subset \mathscr{H}_{\text {as }}:$

$$
\begin{aligned}
\left.\left|\Psi_{\alpha}^{\mathrm{KF}}\right\rangle\right\rangle & :=D_{\tilde{g}_{\alpha}}^{\dagger}\left|\Phi_{\alpha}\right\rangle, \\
D_{\tilde{g}_{\alpha}} & :=\exp \left[\sum_{\tilde{h}} \int d^{3} k\left[g_{\alpha}^{\mu}(\vec{k}) \epsilon_{\mu}^{*}(\vec{k}, \tilde{h}) a^{\dagger}(\vec{k}, \tilde{h})-(\text { H.c. })\right]\right],
\end{aligned}
$$

$$
g_{\alpha}^{\mu}(\vec{k}):=\sum_{n \in \alpha} \frac{\phi^{n}\left(k, p_{n}\right) e_{n}}{(2 \pi)^{3 / 2} \sqrt{2 \omega}}\left(\frac{p_{n}^{\mu}}{p_{n} \cdot k}+c^{\mu}\right)
$$

Here, $\phi^{n}\left(k, p_{n}\right)$ is an arbitrary function as long as the function satisfies the following convergence conditions:

$$
\begin{gathered}
-\frac{1}{2} \sum_{\tilde{h}} \int d^{3} k\left|\tilde{g}_{\alpha}(\vec{k}, \tilde{h})-\tilde{f}_{\alpha}(k, \tilde{h} ; t)\right|^{2}<\infty, \\
\operatorname{Im}\left[\sum_{\tilde{h}} \int d^{3} k \tilde{g}_{\alpha}^{*}(\vec{k}, \tilde{h}) \tilde{f}_{\alpha}(k, \tilde{h} ; t)\right]<\infty,
\end{gathered}
$$

which ensure that $\left.\left|\Psi_{\alpha}^{\mathrm{KF}}\right\rangle\right\rangle$ is in $\mathscr{H}_{\mathrm{as}}$, are satisfied. To satisfy these conditions, $\phi^{n}\left(k, p_{n}\right)$ should satisfy $\phi^{n}\left(k, p_{n}\right)=1$ in the neighborhood of $\omega=0$. We also use the expression $\tilde{g}_{\alpha}(k, \tilde{h}):=g_{\alpha}^{\mu}(\vec{k}) \epsilon_{\mu}^{*}(\vec{k}, \tilde{h})$, and the null vector $c^{\mu}$ satisfies $^{11}$ $c \cdot k=-1$. The null vector $c_{\mu}$ is introduced to dictate their asymptotic state $\left.\left|\Psi_{\alpha}^{\mathrm{KF}}\right\rangle\right\rangle$ to satisfy the free Guputa-Bleuler (GB) condition

$$
\left.k^{\mu} \epsilon_{\mu}(\vec{k}, \tilde{h}) a(\vec{k}, \tilde{h})\left|\Psi_{\alpha}^{\mathrm{KF}}\right\rangle\right\rangle=0 \quad \text { for any } \vec{k}, \tilde{h}
$$

for excluding the scalar mode and longitudinal mode. The free GB condition leads to the condition $k_{\mu} g_{\alpha}^{\mu}(\vec{k})=0$ for the $g_{\alpha}^{\mu}(\vec{k})$ in Eq. (2.32), and we get $g_{\alpha}^{\mu}(\vec{k})$ in Eq. (2.33). Another feature is that Kullish and Faddeev formally have eliminated time dependence by using $\tilde{g}_{\alpha}$ instead of $\tilde{f}_{\alpha}(t)$.

Kulish and Faddeev have defined their $S$-matrix $S_{\beta \alpha}^{\mathrm{KF}}$ as

$$
\begin{aligned}
S_{\beta \alpha}^{\mathrm{KF}} & =\lim _{t^{\prime} \rightarrow \infty, t \rightarrow-\infty}\left\langle\left\langle\Psi_{\beta}^{\mathrm{KF}}\left|\mathcal{S}_{\mathrm{KF}}^{\mathrm{as}}\left(t^{\prime}, t\right)\right| \Psi_{\alpha}^{\mathrm{KF}}\right\rangle\right\rangle \\
& =\lim _{t^{\prime} \rightarrow \infty, t \rightarrow-\infty}\left\langle\Phi_{\beta}\left|D_{\tilde{g}_{\alpha}} \tilde{Z}^{\dagger}\left(t^{\prime}\right) \mathcal{S}\left(t^{\prime}, t\right) \tilde{Z}(t) D_{\tilde{g}_{\alpha}}^{\dagger}\right| \Phi_{\alpha}\right\rangle .
\end{aligned}
$$

\footnotetext{
${ }^{11}$ We use the spacetime metric with signature $(-,+,+,+)$, and we can define $c^{\mu}:=\frac{1}{2 \omega}(1,-\hat{\vec{k}})$. Here, vectors with a hat ( $)$ denote unit vectors like $\hat{\vec{k}}=\vec{k} /|\vec{k}|$.
}

If we can take the limit $|t| \rightarrow \infty$ in advance, $S_{\beta \alpha}^{\mathrm{KF}}$ is formally written by

$$
\begin{aligned}
S_{\beta \alpha}^{\mathrm{KF}} \stackrel{!}{=}\left\langle\left\langle\Psi_{\beta}^{\mathrm{KF}}\left|\mathrm{e}^{-i \hat{\theta}(\infty)} \mathcal{S}_{\mathrm{D}} \mathrm{e}^{i \hat{\theta}(-\infty)}\right| \Psi_{\alpha}^{\mathrm{KF}}\right\rangle\right\rangle \\
=\left\langle\Phi_{\beta}\left|\mathrm{e}^{-i \hat{\theta}(\infty)} D_{\tilde{g}_{\alpha}} \mathcal{S}_{\mathrm{D}} D_{\tilde{g}_{\alpha}}^{\dagger} \mathrm{e}^{i \hat{\theta}(-\infty)}\right| \Phi_{\alpha}\right\rangle .
\end{aligned}
$$

As we will see later, infinite large phase factors in Eq. (2.37) cancel with the infinite large phase factor coming from the soft-photon loop corrections. This cancellation is what we can expect, as in the Dollard formalism. The KF $S$-matrix Eq. (2.37) is accepted as an IR-finite $S$-matrix, but their explanation is inadequate, as we will see in the next section.

\section{Some issues}

Chung has proved that the absolute value of the $S$-matrix is IR finite when we consider the asymptotic state given by

$$
\begin{aligned}
\left.\left|\Psi_{\alpha}^{\mathrm{Ch}}\right\rangle\right\rangle:=\exp \left[\sum _ { h = \pm } \int d ^ { 3 } k \left[f_{\alpha}(\vec{k}, h ; t=0) a^{\dagger}(\vec{k}, h)\right.\right. \\
-(\text { H.c. })]]\left|\Phi_{\alpha}\right\rangle=D_{f_{\alpha}(t=0)}\left|\Phi_{\alpha}\right\rangle, \\
f_{\alpha}(\vec{k}, h ; t):=\sum_{n \in \alpha} \frac{e_{n}}{(2 \pi)^{3 / 2} \sqrt{2 \omega}} \frac{p_{n} \cdot \epsilon^{*}(k, h)}{k \cdot p_{n}} \mathrm{e}^{-i k \cdot v_{n} t} .
\end{aligned}
$$

In other words, $\left|\left\langle\left\langle\Psi_{\beta}^{\mathrm{Ch}}\left|\mathcal{S}_{\mathrm{D}}\right| \Psi_{\alpha}^{\mathrm{Ch}}\right\rangle\right\rangle\right|$ is IR-divergent free [13]. Following this fact, Kulish and Faddeev have concluded that their $S$-matrix Eq. (2.37) is IR finite, because they have considered that their asymptotic state $\left.\left|\Psi_{\alpha}^{\mathrm{KF}}\right\rangle\right\rangle$ of Eq. (2.32) is equivalent to the Chung state $\left.\left|\Psi_{\alpha}^{\mathrm{Ch}}\right\rangle\right\rangle$ of Eq. (2.38) in the IR region. As a matter of fact, however, $\left.\left|\Psi_{\alpha}^{\mathrm{KF}}\right\rangle\right\rangle$ is not equivalent to $\left.\left|\Psi_{\alpha}^{\mathrm{Ch}}\right\rangle\right\rangle$. In the discussion of equivalence, Kulish and Faddeev decomposed $g_{\alpha}^{\mu}(\vec{k})$ as $g_{\alpha}^{\mu}(\vec{k})=g_{\alpha}^{+}(\vec{k}) \epsilon^{\mu}(\vec{k},+)+$ $g_{\alpha}^{-}(\vec{k}) \epsilon^{\mu}(\vec{k},-)$ and regarded $\left.\left|\Psi_{\alpha}^{\mathrm{KF}}\right\rangle\right\rangle$ as a state without unphysical photon modes. However, we should decompose $g_{\alpha}^{\mu}(\vec{k})=g_{\alpha}^{+}(\vec{k}) \epsilon^{\mu}(\vec{k},+)+g_{\alpha}^{-}(\vec{k}) \epsilon^{\mu}(\vec{k},-)+g_{\alpha}^{0}(\vec{k}) k^{\mu}$, since adding the third term also holds the condition $g_{\alpha}^{\mu} k_{\mu}=0$. In this case, we cannot eliminate the unphysical photon modes in $\left.\left|\Psi_{\alpha}^{\mathrm{KF}}\right\rangle\right\rangle$. In other words, the IR divergence of the KF $S$-matrix in Eq. (2.37) is nontrivial in their discussion due to the existence of the unphysical photon modes.

In addition, there is a problem with the free GB condition Eq. (2.35) that Kulish and Faddeev have imposed on their asymptotic state. Usually, the free GB condition is imposed on free-particle states. Is it really appropriate to impose the free GB condition on a state in the presence of asymptotic interactions? Hirai and Sugishita have explored the asymptotic states in QED from the viewpoint of the BRS quantization with asymptotic interaction, and they have concluded that the gauge-invariant condition that prohibits unphysical states in QED is different from the free GB condition, but the condition is given as follows [21]: 


$$
\begin{gathered}
\left.\lim _{|t| \rightarrow \infty} \hat{G}(\vec{k}, \tilde{h}, t)\left|\Psi^{\mathrm{as}}\right\rangle\right\rangle=0 \quad \text { for any } \vec{k} \text { and } \tilde{h}, \\
\hat{G}(\vec{k}, \tilde{h}, t):=k^{\mu} \epsilon_{\mu} a(\vec{k}, \tilde{h})-\hat{q}(\vec{k}, t) \\
\hat{q}(\vec{k}, t):=\int d^{3} p \frac{e \hat{\rho}(\vec{p}) \mathrm{e}^{-i k \cdot v_{p} t}}{(2 \pi)^{3 / 2} \sqrt{2 \omega}}
\end{gathered}
$$

The term $\hat{q}$ has usually been ignored under the assumption that interaction can be neglected in the far past or far future. Although $\left.\left|\Psi_{\alpha}^{\mathrm{KF}}\right\rangle\right\rangle$ does not satisfy this condition when there exists any charged particle in the state, the state

$$
\left.\left|\Psi_{\alpha} \mathrm{KF}^{\prime}(t)\right\rangle\right\rangle:=D_{\tilde{f}_{\alpha}(t)}\left|\Phi_{\alpha}\right\rangle \quad \text { up to phase, }
$$

which is often called the KF state, satisfies the gaugeinvariant condition in Eq. (2.40). ${ }^{12}$ For instance, we can get $\left.\left|\Psi_{\alpha}^{\text {KKF" }}(t)\right\rangle\right\rangle$ by omitting $c_{\mu}$ in $g_{\alpha}^{\mu}$ and by setting $\phi^{n}\left(k, p_{n}\right)=-\mathrm{e}^{-i k \cdot v_{n} t}$, though we come across time dependence of the state. In that sense, Hirai and Sugishita have concluded that the free GB condition is not appropriate for the gauge-invariance condition of the physical asymptotic states, and we do not need $c^{\mu}$. Here, we note that the asymptotic state of Eq. (2.40) is a coherent state with unphysical photon modes. Hence, we cannot apply Chung's arguments straightforwardly from the viewpoint of only the ghost-free condition [Eq. (2.40)]. We also note that we cannot discuss the infinitely large phase factor messing the $S$-matrix ill-defined only by using the ghostfree condition [Eq. (2.40)]. ${ }^{13}$

There are some other nontrivial points. First of all, while Kulish and Faddeev have defined the asymptotic state as a form where we set $\omega=0$, the validity of applying this form to the entire photon-energy region needs to be discussed. In this treatment, we also need to discuss how to deal with the UV divergence from the asymptotic state. In addition, while we derive the dressed operator from the asymptotic interaction, Kulish and Faddeev have removed the contribution from the lower end of the time integral by hand. This treatment is also a bit arbitrary. Also, the validity of taking the limit $|t| \rightarrow \infty$ first and performing the calculation as in Eq. (2.37) is questionable. This is because, although the KF asymptotic state is formally time independent, the convergence conditions in Eq. (2.34) implicitly include time. If we take the limit $|t| \rightarrow \infty$, then we get $\tilde{f}_{\alpha}(t) \rightarrow 0$ from Eq. (2.23). So, $\phi^{n}\left(k, p_{n}\right)$ must be zero to satisfy the convergence conditions. In other words, the dressed state does not appear, and we are back to the

\footnotetext{
${ }^{12}$ The asymptotic state $\mid \Psi_{\alpha}$ "KF" $\left.\left.(t)\right\rangle\right\rangle$ differs from the original KF asymptotic state $\left.\left|\Psi_{\alpha}^{\mathrm{KF}}\right\rangle\right\rangle$ in the Hilbert space to which it belongs. That is, $\mid \Psi_{\alpha}$ "KF" $\left.\left.(t)\right\rangle\right\rangle \notin \mathscr{H}_{\text {as }}$ in general [cf. Eq. (2.25)].

${ }^{13}$ Later, based on the discussion of asymptotic symmetry, Hirai and Sugishita have suggested a candidate of the asymptotic state in QED, which is expected to remove the IR divergence of the $S$-matrix [26].
}

$S$-matrix of the Fock basis [Eq. (2.4)]. Another problem besides the construction method of the asymptotic state is that the KF formalism does not have predictability due to the existence of the functional ambiguity $\operatorname{as}^{14} \phi^{n}\left(k, p_{n}\right)$.

\section{ASYMPTOTIC STATES AND THE $S$-MATRIX}

Asymptotic states are dressed by unphysical photon modes when we consider manifestly covariant QED by adding a gauge-fixing term as in the papers by Kulish and Faddeev or Hirai and Sugishita. This situation causes the discussion of the IR divergent problem to be complicated. The existence of ghosts and unphysical photon modes complicates the structure of the Hilbert space and the construction of asymptotic states by generating debates about the existence of the null vector $c_{\mu}$ and the existence of functional degrees of freedom like $\phi^{n}\left(k, p_{n}\right)$. When approximation regarding an asymptotic state as a freeparticle state is good, we can naturally determine the form of the corresponding suitable asymptotic state from the free Hamiltonian of the theory. ${ }^{15}$ If we can directly determine the asymptotic state from the asymptotic Hamiltonian $H_{\text {as }}$ of the theory without any discussion about the Hilbert space, we may be able to construct the dressed-state formalism with predictability without suffering from functional degrees of freedom. In line with this idea, we discuss the gauge field in the Coulomb gauge, which naturally emerges from the representation theory of massless helicity for one particle. We have the following Lagrangian as a starting point:

$$
\mathcal{L}=-\frac{1}{4} F_{\mu \nu} F^{\mu \nu}-\bar{\Psi}\left(\gamma^{\mu} \partial_{\mu}+m\right) \Psi-i e \bar{\Psi} \gamma^{\mu} \Psi A_{\mu}+\mathcal{L}_{\mathrm{c}}
$$

Here, $\mathcal{L}_{\mathrm{c}}$ is the counterterm for the renormalization. The gauge field $a_{\mu}(x):=\Omega^{\dagger}(t) A_{\mu}(x) \Omega(t)$ in the interaction picture is the physical photon field given by

$$
\begin{aligned}
a_{\mu}(x)= & \int \frac{d^{3} k}{(2 \pi)^{3 / 2} \sqrt{2 \omega}} \sum_{h}\left[\epsilon_{\mu}(\vec{k}, h) \mathrm{e}^{i k \cdot x} a(\vec{k}, h)\right. \\
& \left.+\epsilon_{\mu}^{*}(\vec{k}, h) \mathrm{e}^{-i k \cdot x} a^{\dagger}(\vec{k}, h)\right] .
\end{aligned}
$$

Properties of the polarization vector of the photon field are given in Appendix A. In this case, we should consider the Dirac quantization, which simplifies the structure of the Hilbert space by prohibiting unphysical degrees of

\footnotetext{
${ }^{14}$ There is no way to determine the behavior of $\phi^{n}\left(k, p_{n}\right)$ in the far region from $\omega=0$ in the KF formalism. This would undermine the predictability of the theory.

${ }^{15}$ For example, when we consider the scattering of the charged particles in the particle picture, it is often convenient to choose the asymptotic state as $\left|\Phi_{\alpha}\right\rangle=b_{\sigma_{1}}^{\dagger}\left(\vec{p}_{1}\right) \cdots b_{\sigma_{N}}^{\dagger}\left(\vec{p}_{N}\right) d_{s_{1}}^{\dagger}\left(\vec{q}_{1}\right) \cdots$ $d_{s_{M}}^{\dagger}\left(\vec{q}_{M}\right)|0\rangle$.
} 
freedom, though it complicates the discussion of phase space. We will consider the gauge invariance of the theory after the discussion in the Coulomb gauge condition.

In the previous sections, we have seen that the existence of the asymptotic interaction is essential in the dressed-state formalisms. In the traditional calculation of the standard $S$-matrix of Eq. (2.4), we use the standard Fock states as a basis by taking $|t| \rightarrow \infty$ before calculating. However, in case there exists asymptotic interaction, we cannot replace the order of taking the limit. We can now understand that this is the source of the IR divergence. The KF formalism is an attempt to justify the replacement of the ordering of taking the limit by the new definition of the $S$-matrix based on the dressed states containing an infinite number of photons. As pointed out in Sec. II D, however, this justification is questionable due to the implicit time dependence of the convergent conditions [Eq. (2.34)], and we would not be able to have dressed states in the limit of $|t| \rightarrow \infty$. In addition, there are some other points which seem unnatural, such as the fact that the KF formalism also requires arguments on the UV side due to the existence of photons with any momenta and the validity of the boundary condition $\hat{\widetilde{R}}(t=0)$ in the derivation of their dress operator.

In the following, we solve these problems by introducing the timescale $T$ where the "in/out" asymptotic states are defined. Considering the possibility that we cannot neglect the interaction even in the far past/future $t=t_{\mathrm{I} / \mathrm{F}}$, we rewrite the "in" state and "out" state in equivalent forms:

$$
\begin{aligned}
& \left|\Psi_{\alpha}^{+}\right\rangle \equiv \Omega^{\dagger}\left(t_{I}\right) \Omega\left(t_{I}\right) \lim _{t \rightarrow-\infty} \Omega^{\dagger}(t) \Omega(t)\left|\Psi_{\alpha}^{+}\right\rangle, \\
& \left|\Psi_{\beta}^{-}\right\rangle \equiv \Omega^{\dagger}\left(t_{F}\right) \Omega\left(t_{F}\right) \lim _{t^{\prime} \rightarrow \infty} \Omega^{\dagger}\left(t^{\prime}\right) \Omega\left(t^{\prime}\right)\left|\Psi_{\beta}^{-}\right\rangle .
\end{aligned}
$$

Since there is a time-translation invariance in this stage, we can set $t_{I} \equiv-T$ and $t_{F} \equiv T$. Next, by using the correspondence in Eq. (2.2), we obtain

$$
\begin{aligned}
\left|\Psi_{\alpha}^{+}\right\rangle & \simeq \Omega^{\dagger}\left(t_{I}\right) \mathcal{S}\left(t_{I},-\infty\right)\left|\Phi_{\alpha}\right\rangle \\
\mathcal{S}\left(t_{I},-\infty\right) & =\lim _{t \rightarrow-\infty} \mathcal{T} \exp \left[-i \int_{t}^{t_{I}} d \tau V^{\mathrm{I}}(\tau)\right] .
\end{aligned}
$$

To investigate the interaction at $t=t_{I}$, we now substitute the expressions of free fields into $V^{\mathrm{I}}\left(t_{I}\right)$ and examine the coefficients of $t_{I}$ in the exponents. Terms with coefficients of $\mathcal{O}(1)$ vanish due to the high-frequency oscillation or the Riemann-Lebesgue lemma. On the other hand, we may find that some coefficients are order $\mathcal{O}(1 / T)$ in some momenta regions. Then, we expand the terms with such coefficients with $1 / T$ in the above momenta regions, and we define the leading terms as the asymptotic interaction. ${ }^{16}$ This point is

\footnotetext{
${ }^{16}$ In the next section, we show the concrete formulation of the dressed-state formalism in this paper by considering QED as an example.
}

different from the KF formalism, where Kulish and Faddeev have used just a form of the interaction where we set $\omega=0$. Hence, the asymptotic interaction which remains at $t=t_{I}$ is given by

$$
V^{\mathrm{I}}\left(t_{I}\right)=V_{\mathrm{as}}^{\mathrm{I}}\left(t_{I}\right)+o(1 / T) .
$$

Since we have defined the asymptotic interaction as interaction terms at the leading order of $1 / T$, we should neglect higher-order terms in all calculations. ${ }^{17}$ When $t \leq t_{I}, V^{\mathrm{I}}(t)$ is approximately given by $V_{\text {as }}^{\mathrm{I}}(t)$, and the "in" state can be written by

$$
\begin{aligned}
\left|\Psi_{\alpha}^{+}\right\rangle & =\Omega^{\dagger}\left(t_{I}\right) \mathcal{Z}\left(t_{I},-\infty\right)\left|\Phi_{\alpha}\right\rangle+o(1 / T), \\
\mathcal{Z}\left(t_{I},-\infty\right) & :=\lim _{t \rightarrow-\infty} \mathcal{T} \exp \left[-i \int_{t}^{t_{I}} d \tau V_{\text {as }}^{\mathrm{I}}(\tau)\right] .
\end{aligned}
$$

Now, we define the asymptotic state $\left.\left|\Psi_{\alpha}(-T)\right\rangle\right\rangle$ of the "in" state $\left|\Psi_{\alpha}^{+}\right\rangle$in the far past $t=t_{I} \equiv-T$ as follows:

$$
\left.\left|\Psi_{\alpha}(-T)\right\rangle\right\rangle:=\mathcal{Z}(-T,-\infty)\left|\Phi_{\alpha}\right\rangle
$$

We also define the asymptotic state $\left.\left|\Psi_{\beta}(T)\right\rangle\right\rangle$ of the "out" state $\left|\Psi_{\beta}^{-}\right\rangle$in the far future $t=t_{F} \equiv T$ to be the same as the asymptotic "in" state. The $S$-matrix [Eq. (2.1)] coincides with the limit of the following asymptotic $S$-matrix $S_{\beta \alpha}^{\text {as }}(T)$, as we can see from the construction of the asymptotic state in Eq. (3.3):

$$
\begin{gathered}
S_{\beta \alpha}^{\mathrm{as}}(T):=\left\langle\left\langle\Psi_{\beta}(T)|\mathcal{S}(T,-T)| \Psi_{\alpha}(-T)\right\rangle\right\rangle \\
=\left\langle\Phi_{\beta}\left|\mathcal{Z}^{\dagger}(T, \infty) \mathcal{S}(T,-T) \mathcal{Z}(-T,-\infty)\right| \Phi_{\alpha}\right\rangle, \\
S_{\beta \alpha} \equiv \lim _{T \rightarrow \infty} S_{\beta \alpha}^{\mathrm{as}}(T) .
\end{gathered}
$$

If we leave $T$ as finite, the asymptotic $S$-matrix in Eq. (3.8) is what we desire to obtain.

\section{EXAMPLE: QUANTUM ELECTRODYNAMICS IN COULOMB GAUGE}

In this section, we apply dressed-state formalism in this paper to QED as an explicit example.

\footnotetext{
${ }^{17}$ As we will mention in Sec. IV B, we can define the asymptotic interaction as terms up to the $(\mathrm{sub})^{k}$-leading order of $1 / T$ according to

$$
V^{\mathrm{I}}\left(t_{I}\right)=V_{\mathrm{as}}^{\mathrm{I}}\left(t_{I}\right)+V_{\mathrm{as}, \mathrm{SL}}^{\mathrm{I}}\left(t_{I}\right)+\cdots+V_{\mathrm{as}, \mathrm{S}}^{k} \mathrm{~L}^{\mathrm{I}}\left(t_{I}\right)+o(1 / T),
$$

and this definition may make a difference if we leave $T$ as finite. In this case, we should neglect $(\mathrm{sub})^{k+1}$-leading-order or higherorder terms in all calculations. Throughout this paper, we adopt a framework considering only leading-order dressing.
} 


\section{A. Construction of the asymptotic states}

As mentioned in the previous sections, we consider the QED in the Coulomb gauge condition to exclude unphysical states explicitly and discuss gauge invariance later. The Coulomb gauge condition naturally appears from the representation theory of the Poincare group for the massless particle with helicity 1 . Now, by substituting the expressions of the photon field [Eq. (3.2)] and the spinor field [Eq. (2.8)] into the interaction of the QED,

$$
V^{\mathrm{I}}(t)=i e \int d^{3} x a_{\mu}(x) \bar{\psi}(x) \gamma^{\mu} \psi(x),
$$

we obtain almost the same expression of Eq. (2.10) except that there is no tilde. Following the previous sections, we discuss the momentum integration region to derive the asymptotic interaction. We claim that the coefficients of $t$ in the exponents in the third line of Eq. (2.10) cannot be ignored, because if $T$ is sufficiently large such that $1 / T \ll m$, then in the photon momentum region $\omega \leq 1 / T$, we find that

$$
\omega \pm\left(E_{p}-E_{p \pm k}\right)=\omega \pm E_{p}\left[1-\left(1 \pm \frac{2 \vec{k} \cdot \vec{p}+\vec{k}^{2}}{E_{p}^{2}}\right)^{1 / 2}\right]=-k \cdot v_{p}+\mathcal{O}\left(\omega^{2} \sim(1 / T)^{2}\right) .
$$

Assuming that the interaction remains in the momentum region $\omega \leq 1 / T$ only, the asymptotic interaction which is defined by the leading terms of the interaction expanded by $1 / T$ as in Eq. (4.2) is given by

$$
V_{\text {as }}^{\mathrm{I}}\left(t_{I, F}\right)=e \sum_{h} \int_{\omega \leq 1 / T} \frac{d^{3} k}{(2 \pi)^{3 / 2} \sqrt{2 \omega}} \int d^{3} p v_{p}^{\mu}\left[\epsilon_{\mu}(\vec{k}, h) a(\vec{k}, h) \mathrm{e}^{i k \cdot v_{p} t_{I, F}}+(\text { H.c. })\right] \hat{\rho}(\vec{p}) .
$$

This asymptotic interaction [Eq. (4.3)] is different from the asymptotic interaction given by Kulish and Faddeev in Eq. (2.11), because in Eq. (4.3), there are no unphysical photon modes, only soft photons with energy $\omega \leq 1 / T$. Hence, the UV divergence from asymptotic states does not appear in the formulation proposed in this paper. Now, by following the arguments in Sec. II B, we find that the asymptotic operator $\mathcal{Z}\left(t_{I},-\infty\right)$ for time evolution is given by

$$
\begin{gathered}
\mathcal{Z}\left(t_{I},-\infty\right)=\mathrm{e}^{\hat{R}\left(t_{I}\right)} \mathrm{e}^{i \hat{\theta}\left(t_{I}\right)}, \\
\hat{R}(t):=\int_{\omega \leq 1 / T} d^{3} k \int d^{3} p \sum_{h}\left[\hat{f}(p, k, h ; t) a^{\dagger}(\vec{k}, h)-(\text { H.c. })\right], \\
\hat{f}(p, k, h ; t):=\frac{e \hat{\rho}(\vec{p})}{(2 \pi)^{3 / 2} \sqrt{2 \omega}} \frac{p \cdot \epsilon^{*}(k, h)}{k \cdot p} \mathrm{e}^{-i k \cdot v_{p} t}, \\
i \hat{\theta}\left(t_{I}\right):=\lim _{t \rightarrow-\infty} i \frac{\mathrm{e}^{2}}{8 \pi} \int d^{3} p \int d^{3} q \hat{\rho}(\vec{p}) \hat{\rho}(\vec{q}) \frac{p \cdot q}{\sqrt{(p \cdot q)-m^{4}}} \log \left(t_{I} / t\right) .
\end{gathered}
$$

In contrast to the KF formulation, which omits a term depending on the reference time $t=0$ by hand, we get a dress operator [Eq. (4.5)] which is naturally independent on any conditions at $t=0$ by using the Eq. (2.23). In addition, although the phase operator in Eq. (4.6) includes the logarithmic divergence, the divergence can be controlled, because the operator is finite before we take the limit $t \rightarrow-\infty$.

Now, we construct the asymptotic state of the "in" state $\left.\left|\Psi_{\alpha}\left(t_{I}\right)\right\rangle\right\rangle$. When the asymptotic state in the particle picture includes only charged particles as in Eq. (2.26), we obtain

$$
\begin{gathered}
\left.\left|\Psi_{\alpha}\left(t_{I}\right)\right\rangle\right\rangle=\lim _{t \rightarrow-\infty} \exp \left[i \sum_{m, n \in \alpha} \frac{e_{m} e_{n}}{8 \pi \beta_{m n}} \log \left(t_{I} / t\right)\right]\left|\psi_{\alpha}\right\rangle \otimes\left|f_{\alpha}\left(t_{I}\right)\right\rangle, \\
\left|f_{\alpha}\left(t_{I}\right)\right\rangle=D_{f_{\alpha}\left(t_{I}\right)}|0 ; \gamma\rangle, \quad D_{f_{\alpha}\left(t_{I}\right)}:=\exp \left[\sum_{h} \int_{\omega \leq 1 / T} d^{3} k\left[f_{\alpha}\left(k, h ; t_{I}\right) a^{\dagger}(\vec{k}, h)-(\text { H.c. })\right]\right] .
\end{gathered}
$$

Here, $\beta_{m n}:=\sqrt{1-\frac{m^{4}}{\left(p_{m} \cdot p_{n}\right)^{2}}}$ is the relative velocity between the $n$th and $m$ th particles. We note that $f_{\alpha}\left(k, h ; t_{I}\right)$ is identical with Eq. (2.39), which has been proposed in the Chung states. Since the asymptotic states include only soft photons in the formalism in this paper, we can straightforwardly extend the expressions of the state Eq. (4.7) to the cases including hard 
photons in the asymptotic states. ${ }^{18}$ Thus, $\left|f_{\alpha}\left(t_{I}\right)\right\rangle$ is just a coherent state of the soft photons. That is, the asymptotic states in QED are dressed states clinging with an infinite number of soft photons. Similarly, we can obtain $\left.\left|\Psi_{\beta}\left(t_{F}\right)\right\rangle\right\rangle$, which is the asymptotic state of the "out" state.

\section{B. Asymptotic $S$-matrix and the physical transition rate}

From the definition of the asymptotic $S$-matrix in Eq. (3.8), we can rewrite

$$
S_{\beta \alpha}^{\mathrm{as}}(T)=\mathrm{e}^{i \theta_{\beta \alpha}(T, t)}\left\langle 0\left|D_{\hat{f}(T)}^{\dagger} \prod_{m \in \beta} a\left(p_{m}, \sigma_{m}, \mathscr{N}_{m}\right) \mathcal{S}(T,-T) \prod_{n \in \alpha} a^{\dagger}\left(p_{n}, \sigma_{n}, \mathscr{N}_{n}\right) D_{\hat{f}(-T)}\right| 0\right\rangle,
$$

where $\mathscr{N}_{n}$ represents species of the $n$th particle-i.e., photons, electrons, and positrons. The phase factor $i \theta_{\beta \alpha}(T, t) \operatorname{coming}$ from the dressed asymptotic states is given by

$$
\theta_{\beta \alpha}(T, t):=\sum_{\substack{m, n \in \alpha \\ m, n \in \beta}} \frac{e_{m} e_{n}}{8 \pi \beta_{m n}} \log (T / t) .
$$

It diverges in the limit of $t \rightarrow \infty$. Here, we sum up the cases where $m$ and $n$ are both in the initial state $\alpha$ or both in the final state $\beta$. In Eq. (4.9), $\prod_{n \in m} a\left(p_{m}, \sigma_{m}, \mathscr{N}_{m}\right)$ denotes the sequence of the annihilation operators in the final state $\beta$, and $\prod_{n \in \alpha} a^{\dagger}\left(p_{n}, \sigma_{n}, \mathscr{N}_{n}\right)$ denotes the sequence of the creation operators in the initial state $\alpha$. For simplicity of the discussion, we consider the case in which there are no hard photons in the asymptotic states. We can, however, trivially add hard photons in the initial and final states because, in the formalism in this paper, the creation and annihilation operators of hard photons commute with the dress operators. Defining the symbol $\partial$ as any operator which satisfies $\langle 0| \partial=0$, and omitting arguments of momentum and spin, we find

$$
\begin{aligned}
& D_{\hat{f}(T)}^{\dagger} \prod_{m^{\prime}, n^{\prime}} b_{m^{\prime}} d_{n^{\prime}} \mathcal{S}(T,-T) \prod_{m, n} b_{m}^{\dagger} d_{n}^{\dagger} D_{\hat{f}(-T)} \\
& =\prod_{m^{\prime}, n^{\prime}} b_{m^{\prime}} d_{n^{\prime}}\left(\mathcal{S}(T,-T) D_{f_{\beta}(T)}^{\dagger}+\left[D_{f_{\beta}(T)}^{\dagger}, \mathcal{S}(T,-T)\right]\right) D_{f_{\alpha}(-T)} \prod_{m, n} b_{m}^{\dagger} d_{n}^{\dagger} \\
& =\prod_{m^{\prime}, n^{\prime}} b_{m^{\prime}} d_{n^{\prime}} \mathcal{S}(T,-T) \prod_{m, n} b_{m}^{\dagger} d_{n}^{\dagger} D_{f_{\beta}(T)}^{\dagger} D_{f_{\alpha}(-T)}+\prod_{m^{\prime}, n^{\prime}} b_{m^{\prime}} d_{n^{\prime}} \mathfrak{S}(T,-T) \prod_{m, n} b_{m}^{\dagger} d_{n}^{\dagger} D_{f_{\beta}(T)}^{\dagger} D_{f_{\alpha}(-T)}+\partial,
\end{aligned}
$$

where

$$
\begin{aligned}
\mathfrak{S}(T,-T) & :=\mathcal{T} \exp \left[e \int_{-T}^{T} d \tau \int d^{3} x \mathfrak{A}_{\mu}(x) \bar{\psi}(x) \gamma^{\mu} \psi(x)\right] \\
\mathfrak{A}_{\mu}(x) & :=\sum_{n \in \beta} \int_{\omega \leq 1 / T} \frac{e_{n} d^{3} k}{(2 \pi)^{3} 2 \omega} P_{\mu \nu}(\vec{k}) \frac{p_{n}^{\nu}}{k \cdot p_{n}} \mathrm{e}^{-i k \cdot\left(x-v_{n} T\right)} .
\end{aligned}
$$

In the third line, we have used Eqs. (B2) and (B9) in Appendix B. When $T$ is sufficiently large, we can use the approximation

$$
T \frac{\sin \left[\left(E_{\beta}-E_{\alpha}\right) T\right]}{\left(E_{\beta}-E_{\alpha}\right) T} \approx 2 \pi \delta\left(E_{\beta}-E_{\alpha}\right) \Rightarrow\left\langle\Phi_{\beta}|\mathcal{S}(T,-T)| \Phi_{\alpha}\right\rangle \approx S_{\beta \alpha}^{\mathrm{D}} .
$$

Here, $S_{\beta \alpha}^{\mathrm{D}}$ is the standard Fock-based $S$-matrix defined by Eq. (2.4). For the first term in Eq. (4.11), we get

$$
\prod_{m^{\prime}, n^{\prime}} b_{m^{\prime}} d_{n^{\prime}} \mathcal{S}(T,-T) \prod_{m, n} b_{m}^{\dagger} d_{n}^{\dagger} D_{f_{\beta}(T)}^{\dagger} D_{f_{\alpha}(-T)} \approx\left(S_{\beta \alpha}^{\mathrm{D}}+\partial\right) D_{f_{\beta}(T)}^{\dagger} D_{f_{\alpha}(-T)}=S_{\beta \alpha}^{\mathrm{D}} D_{f_{\beta}(T)}^{\dagger} D_{f_{\alpha}(-T)}+\partial
$$

For the second term in Eq. (4.11), there is no photon operator, and therefore we cannot construct a scattering diagram-i.e.,

\footnotetext{
${ }^{18}$ When we include the hard photon, by separating the energy region of the photon as $\omega \leq 1 / T$ and $1 / T<\omega$, we can define $|0 ; \gamma\rangle=:\left|0 ; \gamma_{\omega \leq 1 / T}\right\rangle \oplus\left|0 ; \gamma_{\omega>1 / T}\right\rangle$.
} 


$$
\prod_{m^{\prime}, n^{\prime}} b_{m^{\prime}} d_{n^{\prime}} \mathfrak{S}(T,-T) \prod_{m, n} b_{m}^{\dagger} d_{n}^{\dagger} D_{f_{\beta}(T)}^{\dagger} D_{f_{\alpha}(-T)}=\partial
$$

Combining Eqs. (4.14) and (4.15) with Eq. (4.11), we get

$$
S_{\beta \alpha}^{\mathrm{as}}(T)=\mathrm{e}^{i \theta_{\beta \alpha}(T, t)}\left\langle f_{\beta}(T) \mid f_{\alpha}(-T)\right\rangle S_{\beta \alpha}^{\mathrm{D}} .
$$

This expression is consistent with Eq. (64) in Chung's paper [13]. Dividing the photon energy region of internal lines into the two regions $\omega \in[1 / t, 1 / T]$ and $\omega>1 / T$, where $1 / t$ is the IR cutoff, we obtain the following expression of $S_{\beta \alpha}^{\mathrm{D}}$ [27]:

$$
\begin{aligned}
S_{\beta \alpha}^{\mathrm{D}} & =\exp \left[\frac{1}{2} \sum_{m, n} \frac{e_{m} e_{n} \eta_{m} \eta_{n}}{(2 \pi)^{3}} \int_{1 / t \leq \omega \leq 1 / T} \frac{d^{3} k}{2 \omega} \alpha_{m n}(k)\right] \exp \left[i \sum_{\substack{m, n \in \in \\
m, n \in \beta}} \frac{e_{m} e_{n}}{8 \pi \beta_{m n}} \log \frac{1 / T}{1 / t}\right] S_{\beta \alpha}^{\mathrm{D}}(1 / T), \\
\alpha_{m n}(k) & :=\frac{\left(p_{m} \cdot p_{n}\right)}{\left(p_{m} \cdot k\right)\left(p_{n} \cdot k\right)} .
\end{aligned}
$$

In the first factor, the summation runs over all external lines, and $\eta_{n}$ is the sign factor, with the value +1 for particles in the final state $\beta$ and the value -1 for particles in the initial state $\alpha$. The third factor, $S_{\beta \alpha}^{\mathrm{D}}(1 / T)$, is the $S$-matrix only including the correction from virtual photons with the energy $\omega>1 / T$, and this can be finite by the prescription of the renormalization. We note that the expression of Eq. (4.17) includes only leading-order soft corrections. The problem of the IR divergence is that the phase factor which appears in the second factor in Eq. (4.17) diverges, and the first factor goes to zero when removing the IR cutoff.

Finally, we calculate $\left\langle f_{\beta}(T) \mid f_{\alpha}(-T)\right\rangle$, which is the inner product of the coherent states and given by

$$
\begin{gathered}
\left\langle f_{\beta}(T) \mid f_{\alpha}(-T)\right\rangle=\Upsilon_{\beta \alpha}(T) \mathrm{e}^{i \chi_{\beta \alpha}(T)}, \\
\Upsilon_{\beta \alpha}(T):=\exp \left[-\frac{1}{2} \sum_{h} \int_{1 / t \leq \omega \leq 1 / T} d^{3} k\left|f_{\beta}(k, h ; T)-f_{\alpha}(k, h ;-T)\right|^{2}\right], \\
i \chi_{\beta \alpha}(T):=i \operatorname{Im} \sum_{h} \int_{1 / t \leq \omega \leq 1 / T} d^{3} k f_{\alpha}(k, h ;-T) f_{\beta}^{*}(k, h ; T) .
\end{gathered}
$$

Here, we have used the IR cutoff $1 / t$, again. We begin with the calculation of $\Upsilon_{\beta \alpha}(T)$. We can see that

$$
\sum_{h} \int d^{3} k\left|f_{\beta}(k, h ; T)-f_{\alpha}(k, h ;-T)\right|^{2}=\sum_{h} \int d^{3} k\left(\left|f_{\alpha}(-T)\right|^{2}+\left|f_{\beta}(T)\right|^{2}-2 \operatorname{Re}\left[f_{\alpha}(-T) f_{\beta}^{*}(T)\right]\right) .
$$

Now, we have omitted $k$ and $h$ in the argument of $f$. For the first term on the rhs of Eq. (4.21), we obtain

$$
\begin{gathered}
\sum_{h} \int d^{3} k\left|f_{\alpha}(-T)\right|^{2}=\frac{1}{(2 \pi)^{3}} \int \frac{d^{3} k}{2 \omega}\left[\sum_{m \in \alpha} e_{m}^{2} \gamma_{m m}(k)+\sum_{\substack{m, n \in \alpha \\
m \neq n}} e_{m} e_{n} \gamma_{m n}(k) \cos \left[\left(v_{m}-v_{n}\right) \cdot k T\right]\right], \\
\gamma_{m n}(k):=\alpha_{m n}(k)+\frac{p_{m} \cdot c}{p_{m} \cdot k}+\frac{p_{n} \cdot c}{p_{n} \cdot k} .
\end{gathered}
$$

We note that $c_{\mu}$ is identical with the null vector introduced by Kulish and Faddeev in Eq. (2.33), $c_{\mu}:=\frac{1}{2 \omega}(1,-\hat{\vec{k}})$. For the third term in Eq. (4.21), we also obtain

$$
\sum_{h} \int d^{3} k f_{\alpha}(-T) f_{\beta}^{*}(T)=\sum_{\substack{m \in \alpha \\ n \in \beta}} \frac{e_{m} e_{n}}{(2 \pi)^{3}} \int \frac{d^{3} k}{2 \omega} \mathrm{e}^{i\left(v_{m}+v_{n}\right) \cdot k T} \gamma_{m n}(k) .
$$

Using Eqs. (4.22) and (4.24), we find 


$$
\log \Upsilon_{\beta \alpha}(T)=-\int \frac{d^{3} k}{2 \omega}\left[\sum_{m, n} \frac{\eta_{m} \eta_{n} e_{m} e_{n}}{2(2 \pi)^{3}} \alpha_{m n}(k)-\sum_{m \neq n} \frac{\eta_{m} \eta_{n} e_{m} e_{n}}{2(2 \pi)^{3}} \gamma_{m n}(k)\left\{1-\cos \left[\left(\eta_{m} v_{m}-\eta_{n} v_{n}\right) \cdot k T\right]\right\}\right]
$$

Here, we use electric charge conservation $\sum_{n} \eta_{n} e_{n}=0$, which is justified from the symmetry of the standard $S$-matrix $S_{\beta \alpha}^{\mathrm{D}}$. The first and second terms in Eq. (4.25) are $\mathcal{O}\left(\omega^{-1}\right) \sim \mathcal{O}(T)$ and $\mathcal{O}(\omega T) \sim \mathcal{O}\left(T^{0}\right)$, respectively. Since the asymptotic states incorporate the asymptotic interaction defined by leading terms with $1 / T$, we should take

$$
\log \Upsilon_{\beta \alpha}(T) \stackrel{\mathrm{L}}{=}-\int \frac{d^{3} k}{2 \omega}\left[\sum_{m, n} \frac{\eta_{m} \eta_{n} e_{m} e_{n}}{2(2 \pi)^{3}} \alpha_{m n}(k)\right]
$$

into account as a correction from dressing. Here, the symbol $\stackrel{\mathrm{L}}{=}$ denotes that the leading terms of the lhs are equal to the leading terms of the rhs. We should take the second term in Eq. (4.25) into account when we consider the effect of the subleading asymptotic interaction and subleading soft radiative corrections. Similarly, we find

$$
i \chi_{\beta \alpha}(T)=\frac{i}{2(2 \pi)^{3}} \sum_{\substack{m \in \alpha, n \in \beta \\ m \in \beta, n \in \alpha}} \int \frac{d^{3} k}{2 \omega} e_{m} e_{n} \gamma_{m n}(k) \sin \left[\left(v_{m}+v_{n}\right) \cdot k T\right]
$$

Phase correction from dressing is $i \theta_{\beta \alpha}(T, t)+i \chi_{\beta \alpha}(T)$, and since we can see that in the $\omega$ integrand

$$
\theta_{\beta \alpha}(T, t)=\mathcal{O}(T), \quad \chi_{\beta \alpha}(T)=\mathcal{O}(\omega T) \sim \mathcal{O}\left(T^{0}\right)
$$

the phase factor $i \chi_{\beta \alpha}(T)$ is neglected in this framework. Finally, the leading-corrected asymptotic $S$-matrix $S_{\beta \alpha}^{\text {as,L }}(T)$ is given by

$$
S_{\beta \alpha}^{\mathrm{as}, \mathrm{L}}(T)=S_{\beta \alpha}^{\mathrm{D}}(1 / T)
$$

This expression is divergence free. Therefore, the asymptotic $S$-matrix Eq. (4.29) is well defined as the operator which connects the asymptotic state at $t=-T$ to the asymptotic state at $t=T$. If there is no state with energy $E<1 / T$ in the Hilbert space for the "in" and "out" states except for the vacuum state, we get

$$
\begin{aligned}
\int d \beta S_{\beta \gamma}^{\mathrm{as}, L^{*}}(T) S_{\beta \alpha}^{\mathrm{as}, \mathrm{L}}(T) & =\int d \beta S_{\beta \gamma}^{\mathrm{D}^{*}}(1 / T) S_{\beta \alpha}^{\mathrm{D}}(1 / T)=\int_{E_{\beta}>1 / T} d \beta\left\langle\Phi_{\gamma}\left|\mathcal{S}_{\mathrm{D}}^{\mathrm{hard}}\right| \Phi_{\beta}\right\rangle\left\langle\Phi_{\beta}\left|\mathcal{S}_{\mathrm{D}}^{\mathrm{hard}}\right| \Phi_{\alpha}\right\rangle \\
& =\int_{E_{\beta}>1 / T} d \beta\left\langle\Psi_{\gamma}^{+} \mid \Psi_{\beta}^{-}\right\rangle\left\langle\Psi_{\beta}^{-} \mid \Psi_{\alpha}^{+}\right\rangle=\left\langle\Psi_{\gamma}^{+} \mid \Psi_{\alpha}^{+}\right\rangle=\delta(\gamma-\alpha) .
\end{aligned}
$$

That is, the asymptotic $S$-matrix $S_{\beta \alpha}^{\text {as,L }}(T)$ is unitary. Here, $\mathcal{S}_{\mathrm{D}}^{\text {hard }}$ denotes the Dyson $S$-operator including only particles with energy $E>1 / T$.

At the end of this section, we discuss the transition rate. We define $\Lambda_{\mathrm{D}}$ as the detection-limit energy of a photon-i.e., we can detect a photon with $\omega>\Lambda_{\mathrm{D}}$, and we cannot detect a photon with $\omega \leq \Lambda_{\mathrm{D}}$. We also define $\Lambda$ as the resolution of energy measurement for the "in" and "out" states-i.e., we can notice the soft-photon emissions when the sum of soft-photon energies exceed $\Lambda$. Now, we have two possibilities:

(i) We cannot observe any soft photons: i.e., $1 / T \leq \Lambda_{\mathrm{D}}$.

(ii) We can observe a soft photon: i.e., $1 / T>\Lambda_{\mathrm{D}}$.

For situation (i), we need to consider the cases in which any number of soft photons with $\omega_{i} \in\left[1 / T, \Lambda_{\mathrm{D}}\right]$ are emitted as long as $\sum_{i} \omega_{i}<\Lambda$. Defining $\Gamma_{\beta \alpha}^{\mathrm{D}}(1 / T)$ as the transition rate derived from $S_{\beta \alpha}^{\mathrm{D}}(1 / T)$, the physical transition rate $\Gamma_{\beta \alpha}^{\mathrm{as}, \mathrm{L}}\left(\Lambda_{\mathrm{D}}, \Lambda\right)$ which we should consider to give predictions for the theory is given by

$$
\Gamma_{\beta \alpha}^{\mathrm{as}, \mathrm{L}}\left(\Lambda_{\mathrm{D}}, \Lambda\right)=\Gamma_{\beta \alpha}^{\mathrm{D}}(1 / T) \sum_{N=0}^{\infty} \frac{\left(A_{\beta \alpha}\right)^{N}}{N !} \int_{1 / T \leq \omega_{i} \leq \Lambda_{\mathrm{D}}, \sum_{i} \omega_{i} \leq \Lambda} \prod_{i=1}^{N} \frac{d \omega_{i}}{\omega_{i}}=\mathcal{F}\left(\Lambda_{\mathrm{D}} / \Lambda ; A_{\beta \alpha}\right)\left(\Lambda_{\mathrm{D}} T\right)^{A_{\beta \alpha}} \Gamma_{\beta \alpha}^{\mathrm{D}}(1 / T)
$$




$$
\begin{gathered}
A_{\beta \alpha}:=-\frac{1}{8 \pi} \sum_{m, n} \frac{\eta_{m} \eta_{n} e_{m} e_{n}}{\beta_{m n}} \log \left(\frac{1+\beta_{m n}}{1-\beta_{m n}}\right)>0 \\
\mathcal{F}(x ; A):=\frac{1}{\pi} \int_{-\infty}^{\infty} d u \frac{\sin u}{u} \exp \left[A \int_{0}^{x} \frac{d \omega}{\omega}\left(\mathrm{e}^{i \omega u}-1\right)\right]=1-\frac{A^{2} \Theta(x-1 / 2)}{2} \int_{1-x}^{x} \frac{d \omega}{\omega} \log \left(\frac{x}{1-\omega}\right)+\cdots
\end{gathered}
$$

We note that $\Gamma_{\beta \alpha}^{\mathrm{as}, \mathrm{L}}\left(\Lambda_{\mathrm{D}}, \Lambda\right)$ is $T$ independent, because $\Gamma_{\beta \alpha}^{\mathrm{D}}(1 / T) \propto(1 / T)^{A_{\beta \alpha}}$. We can safely take the limit of $T \rightarrow \infty$ in this case. This transition rate is exactly the same as the transition rate in the conventional Bloch-Nordsieck formalism.

For situation (ii), we cannot take the limit $T \rightarrow \infty$. We assume that $T$ is fixed to be a finite but sufficiently large value. We can rewrite the asymptotic "in" state as

$$
\left.\left|\Psi_{\alpha}(-T)\right\rangle\right\rangle=\lim _{t \rightarrow \infty} \exp \left[i \sum_{m, n \in \alpha} \frac{e_{m} e_{n}}{8 \pi \beta_{m n}} \log (T / t)\right]\left|\psi_{\alpha}\right\rangle \otimes\left[\left|f_{\alpha}^{\text {invisible }}(-T)\right\rangle \oplus\left|f_{\alpha}^{\text {visible }}(-T)\right\rangle\right],
$$

where "invisible" and "visible" mean that we take only into account soft photons with the energy $\omega \in\left[1 / t, \Lambda_{\mathrm{D}}\right]$ and $\omega \in\left[\Lambda_{\mathrm{D}}, 1 / T\right]$, respectively. If we consider the radiative correction from an invisible photon with $\omega \in\left[1 / t, \Lambda_{\mathrm{D}}\right]$ instead of that from a soft photon with $\omega \in[1 / t, 1 / T]$ in the derivation of Eq. (4.17), we get

$$
\begin{aligned}
S_{\beta \alpha}^{\mathrm{D}} & =\exp \left[\frac{1}{2} \sum_{m, n} \frac{e_{m} e_{n} \eta_{m} \eta_{n}}{(2 \pi)^{3}} \int_{1 / t \leq \omega \leq \Lambda_{\mathrm{D}}} \frac{d^{3} k}{2 \omega} \alpha_{m n}(k)\right] \exp \left[i \sum_{\substack{m, n \in \alpha \\
m, n \in \beta}} \frac{e_{m} e_{n}}{8 \pi \beta_{m n}} \log \frac{\Lambda_{\mathrm{D}}}{1 / t}\right] S_{\beta \alpha}^{\mathrm{D}}\left(\Lambda_{\mathrm{D}}\right), \\
S_{\beta \alpha}^{\mathrm{a}, \mathrm{L}}(T) & =\mathrm{e}^{i \theta_{\beta \alpha}(1 / \Lambda, t)+i \theta_{\beta \alpha}\left(T, \Lambda_{\mathrm{D}}\right)}\left\langle f_{\beta}^{\text {invisible }}(T) \mid f_{\alpha}^{\text {invisible }}(-T)\right\rangle\left\langle f_{\beta}^{\mathrm{visible}}(T) \mid f_{\alpha}^{\mathrm{visible}}(-T)\right\rangle S_{\beta \alpha}^{\mathrm{D}} \\
& =\exp \left[-\frac{1}{2} \sum_{m, n} \frac{e_{m} e_{n} \eta_{m} \eta_{n}}{(2 \pi)^{3}} \int_{\Lambda_{\mathrm{D}} \leq \omega \leq 1 / T} \frac{d^{3} k}{2 \omega} \alpha_{m n}(k)\right] \exp \left[i \sum_{\substack{m, n \in \alpha \\
m, n \in \beta}} \frac{e_{m} e_{n}}{8 \pi \beta_{m n}} \log \Lambda_{\mathrm{D}} T\right] S_{\beta \alpha}^{\mathrm{D}}\left(\Lambda_{\mathrm{D}}\right) .
\end{aligned}
$$

Since we also find

$$
\sum_{m, n} \frac{e_{m} e_{n} \eta_{m} \eta_{n}}{(2 \pi)^{3}} \int_{\Lambda_{\mathrm{D}} \leq \omega \leq 1 / T} \frac{d^{3} k}{2 \omega} \alpha_{m n}(k)=\sum_{m, n} \frac{e_{m} e_{n} \eta_{m} \eta_{n}}{8 \pi \beta_{m n}} \log \left(\frac{1+\beta_{m n}}{1-\beta_{m n}}\right) \log \Lambda_{\mathrm{D}} T=A_{\beta \alpha} \log \Lambda_{\mathrm{D}} T,
$$

the physical transition rate $\Gamma_{\beta \alpha}^{\mathrm{as}, \mathrm{L}}(T)$, which we should consider to give predictions for the theory, is given by

$$
\Gamma_{\beta \alpha}^{\mathrm{as}, \mathrm{L}}(T)=\left(\frac{1}{\Lambda_{\mathrm{D}} T}\right)^{A_{\beta \alpha}} \Gamma_{\beta \alpha}^{\mathrm{D}}\left(\Lambda_{\mathrm{D}}\right)
$$

This transition rate is finite as long as $\Lambda_{\mathrm{D}} T$ is finite and $\Lambda_{\mathrm{D}}$ independent, because $\Gamma_{\beta \alpha}^{\mathrm{D}}\left(\Lambda_{\mathrm{D}}\right) \propto\left(\Lambda_{\mathrm{D}}\right)^{A_{\beta \alpha}}$. If the detection limit of the photon detector is improved, we may distinguish the difference between the transition rate $\Gamma_{\beta \alpha}^{\mathrm{as}, \mathrm{L}}(T)$ for the case (i) and the transition rate $\Gamma_{\beta \alpha}^{\mathrm{D}}\left(\Lambda_{\mathrm{D}}\right)$ for the case (ii) in principle.

\section{Gauge invariance and large gauge symmetry}

So far, we have argued about the asymptotic $S$-matrix in the Coulomb gauge. In this section, we discuss gauge invariance. Since $S_{\beta \alpha}^{\mathrm{D}}$ in Eq. (4.29) is nothing but the standard $S$-matrix and is therefore gauge invariant, we only need to discuss the gauge invariance of $\left\langle f_{\beta}(T) \mid f_{\alpha}(-T)\right\rangle$. For the coherent state of the soft photon $\left|f_{\alpha}( \pm T)\right\rangle$, if we consider the gauge transformation ${ }^{19} \epsilon_{\mu}(k, h) \rightarrow \epsilon_{\mu}(k, h)+\varepsilon_{\mp}^{*}(k, h) k_{\mu}$, we find

$$
f_{\alpha}(k, h ; \pm T) \rightarrow f_{\alpha}(k, h \pm T)+\varepsilon_{\alpha}^{\mp}(k, h ; \pm T), \quad \varepsilon_{\alpha}^{\mp}(k, h ; \pm T):=\sum_{m \in \alpha} \frac{e_{m}}{(2 \pi)^{3 / 2} \sqrt{2 \omega}} \varepsilon_{\mp}(k, h) \mathrm{e}^{\mp i k \cdot v_{m} T},
$$

\footnotetext{
${ }^{19}$ We use the complex conjugate of $\varepsilon$ in order to make notation simple.
} 
and the inner product of the coherent states is transformed as

$$
\begin{gathered}
\left\langle f_{\beta}(T) \mid f_{\alpha}(-T)\right\rangle \rightarrow\left\langle f_{\beta}(T) \mid f_{\alpha}(-T)\right\rangle \times\left\langle\varepsilon_{\beta}^{-}(T) \mid \varepsilon_{\alpha}^{+}(-T)\right\rangle \exp \left[\sum_{h} \int_{1 / t \leq \omega \leq 1 / T} d^{3} k Z_{\beta \alpha}(k, h ; T)\right], \\
Z_{\beta \alpha}(k, h ; T):=-\operatorname{Re}\left[\left(f_{\beta}^{*}(T)-f_{\alpha}^{*}(-T)\right)\left(\varepsilon_{\beta}^{-}(T)-\varepsilon_{\alpha}^{+}(-T)\right)\right]+i \operatorname{Im}\left[f_{\alpha}(-T) \varepsilon_{\beta}^{-*}(T)+f_{\beta}^{*}(T) \varepsilon_{\alpha}^{+}(-T)\right] .
\end{gathered}
$$

We choose the function of the gauge transformation $\varepsilon^{ \pm}$to be a square-integrable function in three-dimensional momentum space. Hence, $\varepsilon^{ \pm}$can generally be expanded by using the spherical harmonics $Y_{\ell m}(\hat{\vec{k}})$ as follows:

$$
\varepsilon_{ \pm}(k, h)=\sum_{i=-1}^{\infty} \sum_{m_{\mathrm{i}}=-\ell_{\mathrm{i}}}^{\ell_{\mathrm{i}}} \sum_{\ell_{\mathrm{i}}=0}^{\infty} \omega^{\mathrm{i}} \chi_{\ell_{\mathrm{i}} m_{\mathrm{i}}}^{ \pm(i)}(h) Y_{\ell_{\mathrm{i}} m_{\mathrm{i}}}(\hat{\vec{k}}) .
$$

Now, we would like to consider the IR contribution of $\left\langle\varepsilon_{\beta}^{-}(T) \mid \varepsilon_{\alpha}^{+}(-T)\right\rangle$. We will refer to the gauge transformation [Eq. (4.42)] with $i=-1$,

$$
\varepsilon_{a}(k, h)=\omega^{-1} \xi^{a}(\hat{\vec{k}}, h), \quad \xi^{a}(\hat{\vec{k}}, h)=\sum_{\ell, m} \chi_{\ell m}^{a}(h) Y_{\ell m}(\hat{\vec{k}}) \neq 0, \quad a=\left\{\begin{array}{l}
- \text { for "out" state } \\
+ \text { for "in" state }
\end{array},\right.
$$

as the large gauge transformation in QFT. Considering that large gauge transformation, we get

$$
\begin{aligned}
\left\langle\varepsilon_{\beta}(T) \mid \varepsilon_{\alpha}(-T)\right\rangle & \stackrel{L}{=} \exp \left[-\frac{1}{2(2 \pi)^{3}} \lim _{t \rightarrow \infty} \log \frac{1 / T}{1 / t} \sum_{m, n} \sum_{h} \int d \Omega_{\hat{\vec{k}}}\left(\eta_{m} \eta_{n} e_{m} e_{n} \xi^{a_{m}}(\hat{\vec{k}}, h) \xi^{a_{n}^{*}}(\hat{\vec{k}}, h)\right)\right] \\
& =\exp \left[-\frac{1}{2(2 \pi)^{3}} \lim _{t \rightarrow \infty} \log \frac{1 / T}{1 / t} \sum_{h} \sum_{\ell, m}\left|Q_{-} \chi_{\ell m}^{-}(h)-Q_{+} \chi_{\ell m}^{+}(h)\right|^{2}\right]
\end{aligned}
$$

where $a_{m}$ denotes - for $m \in \beta$ and + for $m \in \alpha$, while $Q_{+}$and $Q_{-}$are the total electric charges of the "in" and "out" states, respectively. Since the logarithm diverges in the limit $t \rightarrow \infty$, the asymptotic $S$-matrix is nonzero only if

$$
Q_{-} \bar{\ell}_{\ell m}(h)=Q_{+} \chi_{\ell m}^{+}(h) \text { for any } h, \ell, m
$$

holds. Conversely, if Eq. (4.45) is true, $Z_{\beta \alpha}(k, h ; T)$ is subleading with $1 / T$, and hence, we can conclude that the asymptotic $S$-matrix is invariant under large gauge transformation. Since $Q_{-}=Q_{+}$due to the symmetry of the standard $S$-matrix $S_{\beta \alpha}^{\mathrm{D}}$, the condition for gauge invariance in Eq. (4.45) requires

$$
\xi^{-}(\hat{\vec{k}}, h)=\xi^{+}(\hat{\vec{k}}, h)
$$

for the functions of the large gauge transformation for "in/out" asymptotic states. These relations correspond to the existence of the large gauge charge in Ref. [7] and their large gauge transformation.

We discuss the asymptotic symmetry of QED in more detail. We rewrite ${ }^{20}$ the state of Eq. (4.7) or the counterpart for the "out" state as $\left.\left|\Psi_{\alpha}^{0}( \pm T)\right\rangle\right\rangle\left|\psi_{\alpha}\right\rangle \otimes\left|f_{\alpha}^{0}( \pm T)\right\rangle$, and we consider the large gauge transformation

$$
\epsilon_{\mu}(k, h) \rightarrow \epsilon_{\mu}(k, h)+\omega^{-1} \xi(\hat{\vec{k}}, h) k_{\mu}, \quad \xi(\hat{\vec{k}}, h) \neq 0
$$

for $\left.\left|\Psi_{\alpha}^{0}( \pm T)\right\rangle\right\rangle$ and obtain the asymptotic state written by $\left.\left|\Psi_{\alpha}^{\xi}( \pm T)\right\rangle\right\rangle \equiv\left|\psi_{\alpha}\right\rangle \otimes\left|f_{\alpha}^{\xi}( \pm T)\right\rangle$. We can show

$$
\left\langle\langle \Psi _ { \beta } ^ { \eta } ( \pm T ) \| \Psi _ { \alpha } ^ { \xi } ( \pm T ) \rangle \stackrel { \mathrm { L } } { = } \left\{\begin{array}{ll}
\delta(\beta-\alpha) & \text { for } \eta(\hat{\vec{k}}, h)=\xi(\hat{\vec{k}}, h) \\
0 & \text { for } \eta(\hat{\vec{k}}, h) \neq \xi(\hat{\vec{k}}, h)
\end{array},\right.\right.
$$

\footnotetext{
${ }^{20}$ When we consider the Hilbert space, we only need to consider the rays (which identify the states that differ only in their phase factors), so we ignore the phase.
} 


$$
\left\langle\left\langle\Psi_{\beta}^{\eta}( \pm T)|| \Psi_{\alpha}^{\xi}(\mp T)\right\rangle\right\rangle^{\underline{L}}\left\{\begin{array}{ll}
\left\langle f_{\alpha}^{\eta}( \pm T) \mid f_{\alpha}^{\xi}(\mp T)\right\rangle \delta(\beta-\alpha)=0 & \text { for } f_{\alpha} \neq 0 \text { or } f_{\beta} \neq 0 \\
1 & \text { for } f_{\alpha}=f_{\beta}=0
\end{array} .\right.
$$

Here, we have used the fact that $\left|\left\langle f_{\alpha}(T) \mid f_{\alpha}(-T)\right\rangle\right|=0$ unless $\alpha$ does not include any charged particle in Eq. (4.49). We can observe that the Hilbert spaces $\mathscr{H}^{+} / \mathscr{H}^{-}$which the asymptotic "in"/"out" state lives in are divided by the function $\xi(\hat{\vec{k}}, h)$ into super-selection sectors. That is, asymptotic states in QED belong to

$$
\begin{aligned}
\mathscr{H}^{+} \equiv \oplus_{\xi} \mathscr{H}_{\xi}^{+}:=\mathscr{H}_{F} \otimes\left(\oplus_{\xi} \mathscr{H}_{\xi}^{\text {soft }}(-T)\right), \quad \text { where }{ }^{\forall} \alpha, \quad\left|\Phi_{\alpha}\right\rangle \in \mathscr{H}_{F}, \quad\left|f_{\alpha}^{\xi}(-T)\right\rangle \in \mathscr{H}_{\xi}^{\text {soft }}(-T), \\
\mathscr{H}^{-} \equiv \oplus_{\xi} \mathscr{H}_{\xi}^{-}:=\mathscr{H}_{F} \otimes\left(\oplus_{\xi} \mathscr{H}_{\xi}^{\text {soft }}(T)\right), \quad \text { where }{ }^{\forall} \alpha, \quad\left|\Phi_{\alpha}\right\rangle \in \mathscr{H}_{F}, \quad\left|f_{\alpha}^{\xi}(T)\right\rangle \in \mathscr{H}_{\xi}^{\text {soft }}(T) .
\end{aligned}
$$

Here, $\mathscr{H}_{F}$ is the Fock space which comprises charged particles and photons with $\omega>1 / T$ (and vacuum). We can also observe

$$
\mathscr{H}_{\xi}^{\text {soft }}( \pm T) \perp \mathscr{H}_{\eta}^{\text {soft }}( \pm T) \quad \text { if } \xi \neq \eta, \quad \mathscr{H}_{\xi}^{\text {soft }}( \pm T) \perp \mathscr{H}_{\eta}^{\text {soft }}(\mp T)
$$

where the symbol $\perp$ denotes that the inner product of any element of the lhs and any element of the rhs is zero. The general asymptotic state containing the degrees of freedom of the large gauge function $\left.\left|\Psi_{\alpha}^{\xi}( \pm T)\right\rangle\right\rangle$ belongs to $\mathscr{H}_{\xi}^{\mp}$-i.e.,

$$
\left|\Psi_{\alpha}^{\xi}( \pm T)\right\rangle \in \mathscr{H}_{\xi}^{\mp}=\mathscr{H}_{\mathrm{F}} \otimes \mathscr{H}_{\xi}^{\text {soft }}( \pm T) .
$$

Now, we can understand that the operator $\mathcal{S}(T,-T)$ is the map from $\mathscr{H}_{\xi}^{+}$to $\mathscr{H}_{\xi}^{-}$, and the asymptotic symmetry in QED appears as the super-selection rule prohibiting the transition $\mathscr{H}_{\xi}^{+} \rightarrow \mathscr{H}_{\eta}^{-}$if $\eta \neq \xi$. This structure of the Hilbert spaces is quite different from the $\mathrm{KF}$ formalism, where $\mathcal{S}(T,-T)$ is defined as the map from $\mathscr{H}_{\mathrm{F}}$ to $\mathscr{H}_{\mathrm{F}}$.

\section{QED memory effect in the dressed-state formalism}

At the end of this section, we discuss the QED memory effect in the dressed-state formalism. We consider the case in which there are no hard photons in the asymptotic states. We can calculate the expectation value of the photon field at $t=T$ as

$$
\begin{aligned}
\left\langle a_{\mu}(\vec{x}, T)\right\rangle & :=\left\langle f_{\beta}(T)\left|a_{\mu}(\vec{x}, T)\right| f_{\beta}(T)\right\rangle \\
& =\int_{\omega \leq 1 / T} \frac{d^{3} k}{(2 \pi)^{3} 2 \omega}\left[\sum_{m \in \beta} \frac{e_{m}}{k \cdot p_{m}} p_{m}^{\nu} P_{\nu \mu}(\vec{k}) \mathrm{e}^{-\vec{k} \cdot\left(\vec{x}-\vec{v}_{m} T\right)}+(\text { c.c. })\right] \\
& =\sum_{m \in \beta} \frac{e_{m}}{(2 \pi)^{3}} \int_{\omega \leq 1 / T} \frac{d^{3} k}{\omega}\left[\frac{p_{m \mu}}{k \cdot p_{m}}+c_{\mu}+\frac{c \cdot p_{m}}{k \cdot p_{m}} k_{\mu}\right] \cos \left\{\vec{k} \cdot\left(\vec{x}-\vec{v}_{m} T\right)\right\} .
\end{aligned}
$$

Then we find that $\left\langle a_{0}(\vec{x}, T)\right\rangle=0$. For simplicity of the discussion, we evaluate the value at $\vec{x}=0$ as $^{21}$

$$
\begin{aligned}
\langle\vec{a}(\overrightarrow{0}, T)\rangle & =-\sum_{m \in \beta} \frac{e_{m} \vec{v}_{m}}{4 \pi^{2}} \int_{0}^{1 / T} d \omega \int_{-1}^{1} d u \frac{\cos \left(\omega T v_{m} u\right)}{1-v_{m} u}=-\sum_{m \in \beta} \frac{e_{m} \hat{\vec{v}}_{m}}{4 \pi^{2} T} G\left(v_{m}\right), \\
G\left(v_{m}\right) & :=\left[\operatorname{Ci}\left(1+v_{m}\right)-\operatorname{Ci}\left(1-v_{m}\right)\right] \sin 1-\left[\operatorname{Si}\left(1+v_{m}\right)-\operatorname{Si}\left(1-v_{m}\right)\right] \cos 1+2 \operatorname{Si}\left(v_{m}\right),
\end{aligned}
$$

where $\operatorname{Ci}(x)$ is the cosine-integral function defined by

$\operatorname{Ci}(x):=-\int_{x}^{\infty} \frac{\cos t}{t} d t=\gamma_{\mathrm{E}}+\log x+\operatorname{Cin}(x), \quad \operatorname{Cin}(x):=\int_{0}^{x} d t \frac{1-\cos t}{t}, \quad \gamma_{\mathrm{E}}$ : Euler-Mascheroni constant,

and $\operatorname{Si}(x)$ is the sine-integral function defined by

\footnotetext{
${ }^{21}$ In order to give predictions for actual experiment/observation, we should evaluate the values at two points like $\vec{x}= \pm \vec{x}_{0}$. A more detailed and realistic analysis of the memory effect is beyond the scope of this paper.
} 


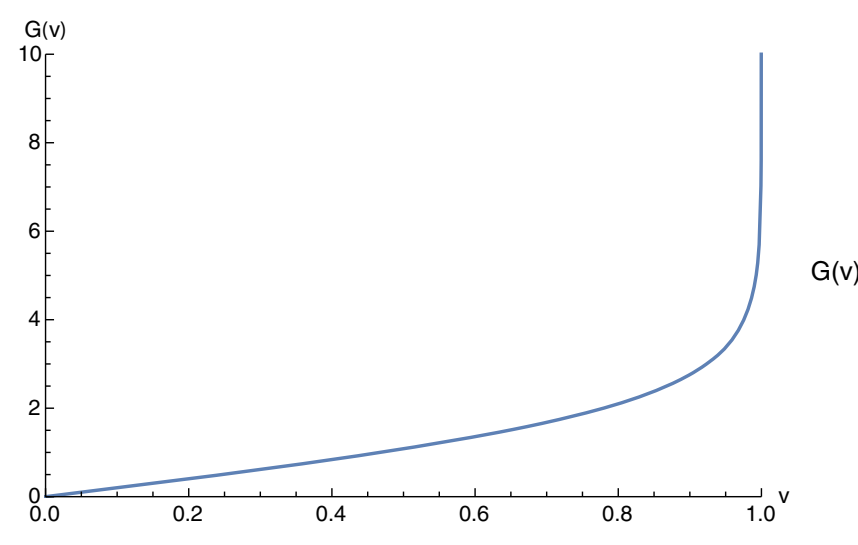

FIG. 1. The behavior of $G(v)$ where $v \in[0,1]$.

$$
\operatorname{Si}(x):=\int_{0}^{x} \frac{\sin t}{t} d t
$$

The behavior of $G(v)$ is depicted in Figs. 1 and 2.

As shown in the figures, $G(v)$ diverges at $v=1$, but even at $v=0.99$, we find $G(v) \sim \mathcal{O}(1)$. Similarly, we can find the expectation value of the vector potential at $x=(-T, \overrightarrow{0})$, and the deviation is given by

$$
\langle\delta \vec{a}(\overrightarrow{0})\rangle=-\sum_{n} \frac{e_{n} \eta_{n} G\left(v_{n}\right)}{4 \pi^{2} T} \hat{\vec{v}}_{n} .
$$

We note that we cannot neglect this memory effect if we take $T$ as finite, because this effect is leading with $1 / T$ in

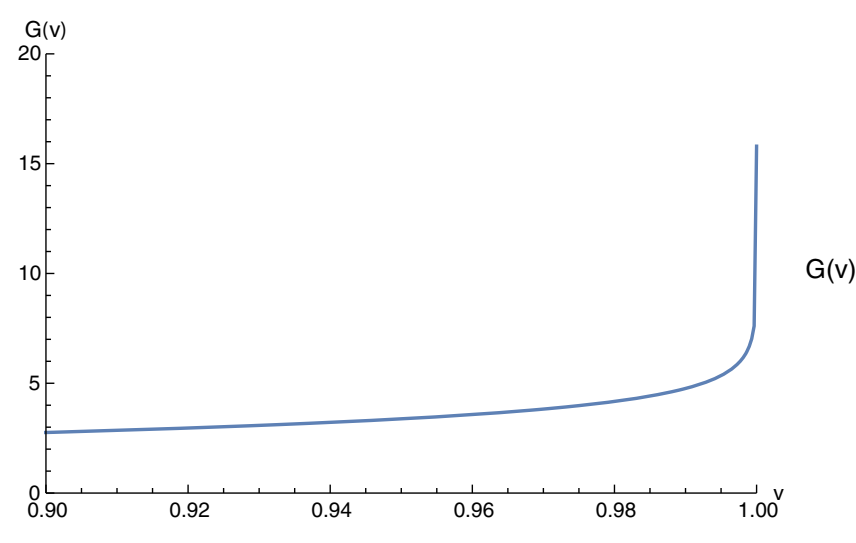

FIG. 2. The behavior of $G(v)$ where $v \in[0.9,1]$.

the calculation for expectation values. We may retrieve some information about the scattering process without observation of the scattering of charged particles by performing the Aharonov-Bohm-effect-like experiments to detect the deviation of the vector potential.

We also comment on the deviation of the electric field and the magnetic field. Although there is a study which discusses the detection of the electromagnetic memory effect by using the deviation of the electromagnetic field in classical theory [28], we find that no deviation appears in both the electric and magnetic fields in the formalism proposed in this paper. For the electric part, this is obvious because we impose the Coulomb gauge condition. For the magnetic part, since $\vec{B}=\nabla \times \vec{a}$, we explicitly find

$$
\langle\delta B(\overrightarrow{0})\rangle=\sum_{m} \frac{e_{m} \eta_{m}}{2(2 \pi)^{3}} \int_{0}^{1 / T} d \omega \omega \int d \Omega_{\vec{k}} \frac{\overrightarrow{\hat{k}} \times \vec{v}_{m}}{1-\overrightarrow{\hat{k}} \cdot \vec{v}_{m}} \sin \left(\omega T \overrightarrow{\hat{k}} \cdot \vec{v}_{m}\right)=0 .
$$

Finally, we discuss the large gauge invariance of the QED memory. We consider the gauge transformation of Eq. (4.47) and expand $\xi(\hat{\vec{k}}, h)$ as follows:

$$
\xi(\hat{\vec{k}}, h)=\sum_{\ell=0}^{\infty} \sum_{m=-\ell}^{\ell} \chi_{\ell m}(h) Y_{\ell m}(\hat{\vec{k}})
$$

Using associated Legendre polynomials $P_{\ell}^{m}(u)$,

$$
P_{\ell}^{m}(u):=2^{-\ell}\left(1-u^{2}\right)^{m / 2} \sum_{j=0}^{\lfloor\ell-m\rfloor} \frac{(-1)^{j}(2 \ell-2 j) !}{j !(\ell-j) !(\ell-2 j-m) !} u^{\ell-2 j-m}
$$

we can express spherical harmonics $Y_{\ell m}(\hat{\vec{k}})$ as

$$
Y_{\ell m}(\theta, \varphi):=C_{\ell, m} s_{m} P_{\ell}^{|m|}(\cos \theta) \mathrm{e}^{i m \varphi}, \quad C_{\ell, m}:=\sqrt{\frac{2 \ell+1}{4 \pi} \frac{(\ell-|m|) !}{(\ell+|m|) !}}, \quad s_{m}:=(-1)^{\frac{m+|m|}{2}} .
$$

Then, we can get the deviation from large gauge transformation $\left\langle\delta_{\varepsilon} \vec{a}(\vec{x})\right\rangle$ as 


$$
\left\langle\delta_{\varepsilon} \vec{a}(\vec{x})\right\rangle=\sum_{n \in \alpha, \beta} \sum_{h} \int_{0}^{1 / T} \frac{e_{n} \eta_{n} d \omega}{(2 \pi)^{3} 2 \sqrt{2}} \sum_{\ell, m} C_{\ell, m} s_{m} \int d \Omega_{\vec{k}} P_{\ell}^{|m|}(\cos \theta)\left[\chi_{\ell m}(h) \mathrm{e}^{i \psi_{m n}}\left(\vec{e}_{\theta}+i h \vec{e}_{\varphi}\right)+(\text { c.c. })\right] .
$$

Here, we have defined

$$
\begin{gathered}
\vec{\epsilon}(\vec{k}, h)=\frac{1}{\sqrt{2}}\left(\vec{e}_{\theta}+i h \vec{e}_{\varphi}\right), \quad \vec{e}_{\theta}:=\left(\begin{array}{c}
\cos \theta \cos \varphi \\
\cos \theta \sin \varphi \\
-\sin \theta
\end{array}\right), \quad \vec{e}_{\varphi}:=\left(\begin{array}{c}
-\sin \varphi \\
\cos \varphi \\
0
\end{array}\right), \\
\psi_{m n}:=m \varphi+\omega \delta x_{n} u, \quad \delta x_{n}:=\left|\vec{x}-\eta_{n} \vec{v}_{n} T\right| .
\end{gathered}
$$

Using the relations

$$
\begin{array}{cc}
\int_{0}^{2 \pi} d \varphi \cos \varphi \cos (a+m \varphi)=\delta_{m \pm 1} \pi \cos a, & \int_{0}^{2 \pi} d \varphi \cos \varphi \sin (a+m \varphi)=\delta_{m \pm 1} \pi \cos a \\
\int_{0}^{2 \pi} d \varphi \sin \varphi \cos (a+m \varphi)=-m \delta_{m \pm 1} \pi \sin a, & \int_{0}^{2 \pi} d \varphi \sin \varphi \sin (a+m \varphi)=m \delta_{m \pm 1} \pi \sin a \\
\int_{0}^{2 \pi} d \varphi \cos (a+m \varphi)=2 \delta_{m 0} \pi \cos a, & \int_{0}^{2 \pi} d \varphi \sin (a+m \varphi)=2 \delta_{m 0} \pi \sin a
\end{array}
$$

and defining

$$
a_{\ell m}(h):=\operatorname{Re}\left[\chi_{\ell m}(h)\right], \quad b_{\ell m}(h):=\operatorname{Im}\left[\chi_{\ell m}(h)\right]
$$

we get

$$
\begin{aligned}
\left\langle\delta_{\varepsilon} \vec{a}(\vec{x})\right\rangle= & \sum_{n \in \alpha, \beta} \sum_{h} \int_{0}^{1 / T} \frac{e_{n} \eta_{n} d \omega}{8 \sqrt{2} \pi^{2}} \sum_{\ell, m} C_{\ell, m} s_{m} \int_{-1}^{1} d u P_{\ell}^{|m|}(u)\left[\left(u \cos \psi_{0 n}+h m \sin \psi_{0 n}\right)\left(a_{\ell m}(h)-b_{\ell m}(h)\right) \delta_{m \pm 1} \vec{e}_{1}\right. \\
& \left.-\left(h \cos \psi_{0 n}+u m \sin \psi_{0 n}\right)\left(a_{\ell m}(h)+b_{\ell m}(h)\right) \delta_{m \pm 1} \vec{e}_{2}-2 \sqrt{1-u^{2}}\left(a_{\ell 0}(h) \cos \psi_{0 n}-b_{\ell 0}(h) \sin \psi_{0 n}\right) \delta_{m 0} \vec{e}_{3}\right] \\
\vec{e}_{1}:= & (1,0,0), \quad \vec{e}_{2}:=(0,1,0), \quad \vec{e}_{3}:=(0,0,1)
\end{aligned}
$$

Considering the fact that the parity of the associated Legendre polynomials is $P_{\ell}^{m}(-u)=(-1)^{m+\ell} P_{\ell}^{m}(u)$, we finally obtain

$$
\begin{aligned}
\left\langle\delta_{\varepsilon} \vec{a}(\vec{x})\right\rangle= & \sum_{n \in \alpha, \beta} \sum_{h} \int_{0}^{1 / T} \frac{e_{n} \eta_{n} d \omega}{8 \sqrt{2} \pi^{2}} \int_{-1}^{1} d u\left[\sum_{k=1}^{\infty} C_{2 k, 1} P_{2 k}^{1}(u) \sum_{m= \pm 1} s_{m} B_{2 k, m}(h)\left(u \cos \psi_{0 n}+h m \sin \psi_{0 n}\right) \vec{e}_{1}\right. \\
& -\sum_{k=1}^{\infty} C_{2 k-1,1} P_{2 k-1}^{1}(u) \sum_{m= \pm 1} s_{m} A_{2 k-1, m}(h)\left(h \cos \psi_{0 n}+u m \sin \psi_{0 n}\right) \vec{e}_{2} \\
& \left.-2 \sqrt{1-u^{2}} \sum_{k=0}^{\infty}\left(C_{2 k, 0} P_{2 k}^{0}(u) a_{2 k, 0}(h) \cos \psi_{0 n}-C_{2 k+1,0} P_{2 k+1}^{0}(u) b_{2 k+1,0}(h) \sin \psi_{0 n}\right) \vec{e}_{3}\right] \\
A_{\ell, m}(h):= & a_{\ell m}(h)+b_{\ell m}(h), \quad B_{\ell, m}(h):=a_{\ell m}(h)-b_{\ell m}(h) .
\end{aligned}
$$

If the QED memory is a large gauge-invariant physical quantity, the last expression in Eq. (4.71) should be identically zero. For the case where the mode $\ell$ is an even number, it leads to the conditions

$$
\begin{aligned}
\operatorname{Re}\left[\chi_{\ell 0}(-h)\right] & =-\operatorname{Re}\left[\chi_{\ell 0}(h)\right] \\
\operatorname{Re}\left[\chi_{\ell \pm 1}(-h)\right]-\operatorname{Im}\left[\chi_{\ell \pm 1}(-h)\right] & =\operatorname{Re}\left[\chi_{\ell \mp 1}(h)\right]-\operatorname{Im}\left[\chi_{\ell \mp 1}(h)\right]
\end{aligned}
$$

and for the case where the mode $\ell$ is an odd number, it leads to the conditions 


$$
\begin{aligned}
\operatorname{Im}\left[\chi_{\ell 0}(-h)\right] & =-\operatorname{Im}\left[\chi_{\ell 0}(h)\right], \\
\operatorname{Re}\left[\chi_{\ell \pm 1}(-h)\right]+\operatorname{Im}\left[\chi_{\ell \pm 1}(-h)\right] & =-\operatorname{Re}\left[\chi_{\ell \mp 1}(h)\right]-\operatorname{Im}\left[\chi_{\ell \mp 1}(h)\right] .
\end{aligned}
$$

Therefore, if and only if the $|m| \leq 1$ modes of the large gauge function $\xi(\hat{\vec{k}}, h)$ satisfy the conditions in Eqs. (4.72) and (4.73), the QED memory is a large gauge-invariant physical quantity. A discussion of the implications of extra conditions to preserve the large gauge symmetry of the QED memory is a subject for future work.

\section{SUMMARY AND PROSPECTS}

In this paper, by starting with the definition of the $S$-matrix, we have proposed a dressed-state formalism to construct the asymptotic state of the "in/out" state in the sufficiently far past/future at $t=\mp T$. In this formalism, we define the asymptotic interaction remaining at $|t|>T$ in terms of some fixed order of $1 / T$ and incorporate it into the asymptotic states. According to the order of $1 / T$ in the asymptotic states, we determine the order of $1 / T$, which we should take into account for all calculations. We can construct the asymptotic states only from the interaction of the theory. ${ }^{22}$ We have also proposed the asymptotic $S$-matrix connecting the asymptotic state of the "in" state at $t=-T$ with the asymptotic state of the "out" state at $t=-T$. We have shown that the asymptotic $S$-matrix is divergence free and unitary, at least in QED. We have also discussed the transition rate, and we have shown that the physical transition rate is exactly the same as the transition rate obtained by the Bloch-Nordsieck formalism when we cannot observe soft photons with $\omega<1 / T$. In this case, we can safely take the limit of $T \rightarrow \infty$. Also, we have seen that the dressed-state formalism in this paper gives us a clear picture of how the relation known as the infrared triangle appears in QFT. For example, we have shown that in QED,

(i) The large gauge transformation corresponds to the gauge transformation for the asymptotic states.

(ii) The large gauge symmetry implies that Hilbert space is divided by the large gauge functions into the super-selection sectors labeled by the functions.

\footnotetext{
${ }^{22} \mathrm{We}$ have considered the interaction of $\mathrm{QED}, V_{\mathrm{QED}}^{\mathrm{I}}(t)$, as

$$
\begin{aligned}
V_{\mathrm{QED}}^{\mathrm{I}}(t) & =V^{\mathrm{I}}(t) \Theta(T-|t|)+V_{\mathrm{as}}^{\mathrm{I}}(t) \Theta(|t|-T), \\
\Theta(x) & =\left\{\begin{array}{ll}
1 & \text { for } x \geq 0 \\
0 & \text { for } x<0
\end{array} .\right.
\end{aligned}
$$
}

Of course, there are other ways to smoothly connect $V^{\mathrm{I}}(t)$ to $V_{\text {as }}^{\mathrm{I}}(t)$ without a step function; however, the difference can affect at most only an $\mathcal{O}(1 / T)$ contribution. On the other hand, as mentioned in a footnote of Sec. II D, the KF formalism has no predictability, since we have no way to decide non-IR behavior of the functional ambiguity $\phi^{n}\left(k, p_{n}\right)$. (iii) The memory effect appears as the deviation of the expectation value of the vector potential caused by soft photons with the information of charged particles.

We may expect that such correspondence also appears in QFTs other than QED. The fact that we can see such correspondences and the fact that we can construct a unitary $S$-matrix may show that the formalism in this paper gives a unified and effective way to understand IR physics.

Furthermore, we have discovered that we can obtain a verifiable prediction in principle by leaving the sufficiently large time that $T$ is finite. Although we will need more detailed discussion, we may verify the deviation of the transition rate if we have a photon detector with a significantly high sensitivity, and we may detect the memory effect in future experiments.

In addition to giving the above perspective, the dressedstate formalism in this paper is different from the KF formalism in the following points:

(1) We do not suffer from the UV divergence from the asymptotic states.

(2) Since the creation and annihilation operators of the soft photon commute with those of the hard photon, we can add hard photons to the asymptotic states straightforwardly.

(3) We do not need to require that the dress operator vanish at $t=0$.

(4) We do not have a functional ambiguity undermining predictability.

We should note that the definitions of the asymptotic states and the asymptotic $S$-matrix proposed in this paper do not depend, at least formally, on the kind of theory. We may expect that the formulation in this paper may also give nontrivial results for theories with massless particles besides QED. For example, some studies indicate that asymptotic symmetry of scalar QED derives the subleading soft-photon theorem $[29,30]$. There are some arguments that there exist asymptotic symmetries besides gauge theory $[31,32]$. Our formulation may give a new way of understanding the asymptotic symmetries from the viewpoint of the asymptotic states in QFT. We may also derive and give a new insight into the subleading (or higher) dressing which has been proposed as a counterpart of the subleading soft theorem [33]. When we leave $T$ as finite, subleading terms may affect the physical transition rate in case (ii) in Sec. IV B and the memory effect. It is also interesting to apply the formulation in this paper to the linearized gravity theory. In previous studies, the $\mathrm{KF}$ formalism or so-called KF state $\left.\left|\Psi_{\alpha}^{\text {"KF" }}(t)\right\rangle\right\rangle$ of Eq. (2.42) is used to study linearized gravity theory $[19,23]$. We may 
construct more suitable asymptotic states for analyzing the IR physics in the formalism in this paper.

Finally, we mention the relation with the information paradox or information loss problem in the BH. In Ref. [12], it is shown that the photons with information on the current having fallen into the $\mathrm{BH}$ appear in the horizon and at null infinity. The authors of Ref. [12] claim that these photons are soft photons generated by the large gauge charges associated with the asymptotic symmetry. In this perspective, they discuss how the asymptotic symmetry and their charges are important for understanding the information paradox. On the other hand, in the analyses in this paper, the asymptotic symmetry appears as just a restriction on the gauge transformation for the soft photons in the asymptotic states-that is, we cannot elicit the information of particles in the initial state from the asymptotic symmetry. Instead, the asymptotic photon states themselves have the information via the function of a softphoton coherent state $f_{\alpha}$. From this viewpoint, we may infer that when we consider the information paradox from the IR physics, the charges are not so important, but the dressed states are essentially important. From different contexts, there have been similar considerations using the dressed states $[20,34,35]$. Indeed, although we can obtain the soft-photon theorem from the asymptotic symmetry, the theorem itself cannot remove the IR divergence in the $S$-matrix. Therefore, we cannot discuss their unitarity. As we have shown, the asymptotic $S$-matrix proposed in this paper is unitary and expected to be a starting point to consider the information paradox. Also, there are some studies to investigate the differences generated by the dressed states $[25,36]$. We expect that research using the dressed states may become more important.

\section{ACKNOWLEDGMENTS}

This work is supported by the JSPS Grant-in-Aid for Scientific Research (C) No. 18K03615 (S. N.).

\section{APPENDIX A: PROPERTIES OF THE COEFFICIENT FUNCTIONS}

The gamma matrix is defined by

$$
\gamma^{\mu}=\left(\gamma^{0}, \vec{\gamma}\right), \quad \gamma^{0}:=-i\left(\begin{array}{cc}
0 & I \\
I & 0
\end{array}\right), \quad \vec{\gamma}:=-i\left(\begin{array}{cc}
0 & \vec{\sigma} \\
-\vec{\sigma} & 0
\end{array}\right)
$$

where $\sigma^{i}$ s are the Pauli matrices

$$
\sigma^{1}:=\left(\begin{array}{cc}
0 & 1 \\
1 & 0
\end{array}\right), \quad \sigma^{2}:=\left(\begin{array}{cc}
0 & -i \\
i & 0
\end{array}\right), \quad \sigma^{3}:=\left(\begin{array}{cc}
1 & 0 \\
0 & -1
\end{array}\right)
$$

We also note that $\beta:=i \gamma^{0}$. In the above definitions, the coefficient functions of the spinor field satisfy the following equations:

$$
\begin{aligned}
\left(i p^{\mu} \gamma_{\mu}+m\right) u_{\sigma}(\vec{p})=0, & \bar{u}_{\sigma}(\vec{p}) u_{\sigma^{\prime}}(\vec{p})=\delta_{\sigma \sigma^{\prime}} \frac{m}{E_{p}}, & \sum_{\sigma} \bar{u}_{\sigma}(\vec{p}) u_{\sigma}(\vec{p})=\frac{1}{2 E_{p}}\left(-i p^{\mu} \gamma_{\mu}+m\right) \beta, \\
\left(i p^{\mu} \gamma_{\mu}-m\right) v(\vec{p}, \sigma)=0, & \bar{v}_{\sigma}(\vec{p}) v_{\sigma^{\prime}}(\vec{p})=-\delta_{\sigma \sigma^{\prime}} \frac{m}{E_{p}}, & \sum_{\sigma} \bar{v}_{\sigma}(\vec{p}) v_{\sigma}(\vec{p})=-\frac{1}{2 E_{p}}\left(i p^{\mu} \gamma_{\mu}+m\right) \beta
\end{aligned}
$$

We define the polarization vectors for the unphysical modes $I=\{S, L\}$ as

$$
\epsilon_{\mu}(\vec{k}, S):=(1, \overrightarrow{0}), \quad \epsilon_{\mu}(\vec{k}, L):=(0, \hat{\vec{k}})
$$

Then we find

$$
\sum_{I} \epsilon_{\mu}^{*}(\vec{k}, I) \epsilon_{\nu}(\vec{k}, I)=-\left(c_{\mu} k_{\nu}+k_{\mu} c_{\nu}\right)=: Q_{\mu \nu}(\vec{k}), \quad c^{\mu}:=\frac{1}{2 \omega}(1,-\hat{\vec{k}})
$$

Since $Q^{2}=Q$ and $Q_{\mu \nu}(\vec{k}) k^{\nu}=k_{\mu}$, this operator is the projection operator to the on-shell momentum direction. The properties of the physical modes $h=\{+,-\}$ are sufficient here if we know the following: 


$$
\epsilon_{0}(\vec{k}, h)=0, \quad \vec{k} \cdot \vec{\epsilon}(\vec{k}, h)=0, \quad \sum_{h} \epsilon_{\mu}^{*}(\vec{k}, h) \epsilon_{\nu}(\vec{k}, h)=\eta_{\mu \nu}-Q_{\mu \nu}(\vec{k})=: P_{\mu \nu}(\vec{k})
$$

Since $P^{2}=P$ and $P_{\mu \nu}(\vec{k}) k^{\nu}=0$, this operator is the projection operator to the transverse direction of the on-shell momentum. We now obtain the following commutation relations:

$$
\left[a_{\mu}(\vec{k}, \tilde{h}), a_{\mu}^{\dagger}\left(\vec{k}^{\prime}, \tilde{h}^{\prime}\right)\right]=\eta_{\mu \nu} \delta_{\tilde{h} \tilde{h}^{\prime}} \delta^{3}\left(\vec{k}-\vec{k}^{\prime}\right), \quad\left[a_{\mu}(\vec{k}, h), a_{\mu}^{\dagger}\left(\vec{k}^{\prime}, h^{\prime}\right)\right]=P_{\mu \nu}(\vec{k}) \delta_{h h^{\prime}} \delta^{3}\left(\vec{k}-\vec{k}^{\prime}\right) .
$$

Since we can calculate the propagator as the vacuum expectation value of the time-ordered product of two fields at points $x$ and $y$, we find that the propagators for the vector field $\tilde{a}_{\mu}$ and the photon field $a_{\mu}$ are given by

$$
\begin{aligned}
& -i \tilde{\Delta}_{\mu \nu}(x-y)=\frac{-i}{(2 \pi)^{4}} \int d^{4} k \frac{\eta_{\mu \nu}}{k^{2}-i \varepsilon} \mathrm{e}^{i k \cdot(x-y)}, \\
& -i \Delta_{\mu \nu}(x-y)=\frac{-i}{(2 \pi)^{4}} \int d^{4} k \frac{\tilde{P}_{\mu \nu}(k)}{k^{2}-i \varepsilon} \mathrm{e}^{i k \cdot(x-y)},
\end{aligned}
$$

where $\tilde{P}_{\mu \nu}(k)$ is the projection operator extended to the off-shell momentum as ${ }^{23}$

$$
\tilde{P}_{\mu \nu}(k)=\eta_{\mu \nu}-\tilde{Q}_{\mu \nu}(k), \quad \tilde{Q}_{\mu \nu}(k)=\left(k_{\mu} k_{\nu}-k^{0} k_{\mu} t_{\nu}-k^{0} t_{\mu} k_{\nu}-k^{2} t_{\mu} t_{\nu}\right) /\left|\vec{k}^{2}\right|, \quad t^{\mu}=(1,0,0,0) .
$$

Although the expression of Eq. (A9) is not Lorentz covariant, we can use an effective propagator as in Eq. (A8) for calculations in the theory with the Lagrangian of Eq. (2.6), because the noncovariant term of the propagator can be eliminated by the noncovariant term in the Hamiltonian.

\section{APPENDIX B: PROPERTIES OF THE COHERENT STATE IN QUANTUM MECHANICS}

Using the Baker-Campbell-Hausdorff formula

$$
\begin{aligned}
\mathrm{e}^{A} \mathrm{e}^{B}= & \exp \left[A+B+\frac{1}{2}[A, B]+\frac{1}{12}[A,[A, B]]\right. \\
& \left.-\frac{1}{12}[B,[A, B]]+\cdots\right]
\end{aligned}
$$

we can reorder the displacement operator $D_{\alpha}$ as

$$
D_{\alpha}:=\exp \left[\alpha a^{\dagger}-\alpha^{*} a\right]=\mathrm{e}^{-\frac{1}{2}|\alpha|^{2}} \mathrm{e}^{\alpha a^{\dagger}} \mathrm{e}^{-\alpha^{*} a}
$$

The coherent state $|\alpha\rangle$ with a parameter $\alpha \in \mathbb{C}$ is given by

$$
|\alpha\rangle:=D_{\alpha}|0\rangle=\mathrm{e}^{-\frac{1}{2}|\alpha|^{2}} \mathrm{e}^{\alpha a}|0\rangle .
$$

Using the relation

\footnotetext{
${ }^{23}$ We obtain this off-shell projection operator more explicitly when we rewrite Eq. (A5) as $Q_{\mu \nu}=\hat{\vec{k}}_{\mu} \hat{\vec{k}}_{\nu}-\hat{\vec{k}}_{\mu} t_{\nu}-t_{\mu} \hat{\vec{k}}_{\nu}$.
}

$$
\left[a, f\left(a^{\dagger}, a\right)\right]=\frac{\partial}{\partial a^{\dagger}} f\left(a^{\dagger}, a\right),
$$

we can easily find

$$
a|\alpha\rangle=\left[a, D_{\alpha}\right]|0\rangle=\alpha|0\rangle ;
$$

i.e., $|\alpha\rangle$ is the eigenstate of the annihilation operator with the eigenvalue $\alpha$. With the particle number bases $|n\rangle$ given by

$$
|n\rangle:=\frac{1}{\sqrt{n !}}\left(a^{\dagger}\right)^{n}|0\rangle,
$$

we can rewrite $|\alpha\rangle$ as

$$
|\alpha\rangle=\mathrm{e}^{-\frac{1}{2}|\alpha|^{2}} \sum_{n=0}^{\infty} \frac{\alpha^{n}}{\sqrt{n !}}|n\rangle .
$$

Thus, the coherent state is a superposition of any number of the particles. The expectation value of the number of the particles is given by

$$
\left\langle\alpha\left|a^{\dagger} a\right| \alpha\right\rangle=|\alpha|^{2} .
$$

In the dressed states of QFT, the expectation value of the particle number is infinite due to the IR divergence of the counterpart of $|\alpha|^{2}$. It is frequently convenient to use the relations

$$
\begin{aligned}
{\left[\mathrm{e}^{a},\left(a^{\dagger}\right)^{n}\right] } & =\sum_{k=0}^{n-1}{ }_{n} \mathrm{C}_{n-k}\left(a^{\dagger}\right)^{k} \mathrm{e}^{a}, \\
{\left[a^{n}, \mathrm{e}^{a^{\dagger}}\right] } & =\mathrm{e}^{a^{\dagger}} \sum_{k=0}^{n-1}{ }_{n} \mathrm{C}_{n-k} a^{k} .
\end{aligned}
$$


The inner product of coherent states is given by

$$
\langle\beta \mid \alpha\rangle=\mathrm{e}^{-\frac{1}{2}\left(|\alpha|^{2}+|\beta|^{2}\right)}\left\langle 0\left|\mathrm{e}^{\beta^{*} a} \mathrm{e}^{\alpha a^{\dagger}}\right| 0\right\rangle=\mathrm{e}^{-\frac{1}{2}\left(|\alpha|^{2}+|\beta|^{2}\right)} \sum_{n=0}^{\infty} \frac{\left(\alpha \beta^{*}\right)^{n}}{n !}\left\langle 0\left|\mathrm{e}^{\alpha a^{\dagger}}\right| 0\right\rangle=\mathrm{e}^{-\frac{1}{2}\left(|\alpha|^{2}+|\beta|^{2}\right)} \mathrm{e}^{\alpha \beta^{*}} .
$$

Or, equivalently,

$$
\langle\beta \mid \alpha\rangle=\mathrm{e}^{-\frac{1}{2}|\beta-\alpha|^{2}} \mathrm{e}^{i \operatorname{Im} \alpha \beta^{*}} .
$$

Thus, coherent states satisfy the normality condition $\langle\alpha \mid \alpha\rangle=1$ but do not satisfy the orthogonality condition, because $\langle\beta \mid \alpha\rangle \neq 0$ in general. In QFT, however, the orthogonality often appears, because the counterpart of $|\beta-\alpha|^{2}$ can be divergent. The inner product of the parameter-translated coherent state is given by

$$
\langle\beta+\eta \mid \alpha+\xi\rangle=\langle\beta \mid \alpha\rangle\langle\eta \mid \xi\rangle \exp \left[-\operatorname{Re}\left[(\beta-\alpha)\left(\eta^{*}-\xi^{*}\right)\right]+i \operatorname{Im}\left[\alpha \eta^{*}+\xi \beta^{*}\right]\right] .
$$

[1] F. Bloch and A. Nordsieck, Phys. Rev. 52, 54 (1937).

[2] D. R. Yennie, S. C. Frautschi, and H. Suura, Ann. Phys. (N.Y.) 13, 379 (1961).

[3] S. Weinberg, Phys. Rev. 140, B516 (1965).

[4] A. Strominger, J. High Energy Phys. 07 (2014) 151.

[5] T. He, P. Mitra, A. P. Porfyriadis, and A. Strominger, J. High Energy Phys. 10 (2014) 112.

[6] M. Campiglia and A. Laddha, J. High Energy Phys. 07 (2015) 115.

[7] D. Kapec, M. Pate, and A. Strominger, Adv. Theor. Math. Phys. 21, 1769 (2017).

[8] A. Strominger, arXiv:1703.05448.

[9] H. Bondi, Nature (London) 186, 535 (1960).

[10] R. K. Sachs, Proc. R. Soc. A 270, 103 (1962).

[11] T. He, V. Lysov, P. Mitra, and A. Strominger, J. High Energy Phys. 05 (2015) 151.

[12] S. W. Hawking, M. J. Perry, and A. Strominger, Phys. Rev. Lett. 116, 231301 (2016).

[13] V. Chung, Phys. Rev. 140, B1110 (1965).

[14] T. W. B. Kibble, J. Math. Phys. (N.Y.) 9, 315 (1968).

[15] T. W. B. Kibble, Phys. Rev. 173, 1527 (1968).

[16] T. W. B. Kibble, Phys. Rev. 174, 1882 (1968).

[17] T. W. B. Kibble, Phys. Rev. 175, 1624 (1968).

[18] P. P. Kulish and L. D. Faddeev, Theor. Math. Phys. 4, 745 (1970).

[19] J. Ware, R. Saotome, and R. Akhoury, J. High Energy Phys. 10 (2013) 159.

[20] D. Neuenfeld, arXiv:1810.11477.

[21] H. Hirai and S. Sugishita, J. High Energy Phys. 06 (2019) 023.
[22] D. Kapec, M. Perry, A. M. Raclariu, and A. Strominger, Phys. Rev. D 96, 085002 (2017).

[23] S. Choi and R. Akhoury, J. High Energy Phys. 02 (2018) 171.

[24] J. D. Dollard, J. Math. Phys. (N.Y.) 5, 729 (1964).

[25] D. Carney, L. Chaurette, D. Neuenfeld, and G. W. Semenoff, Phys. Rev. D 97, 025007 (2018).

[26] H. Hirai and S. Sugishita, J. High Energy Phys. 02 (2021) 025.

[27] S. Weinberg, The Quantum Theory of Fields (Cambridge University Press, Cambridge, 1996).

[28] Y. Hamada and S. Sugishita, J. High Energy Phys. 07 (2018) 017.

[29] H. Hirai and S. Sugishita, J. High Energy Phys. 07 (2018) 122.

[30] M. Campiglia and A. Laddha, J. High Energy Phys. 10 (2019) 287.

[31] M. Campiglia, L. Coito, and S. Mizera, Phys. Rev. D 97, 046002 (2018).

[32] Y. Hamada and S. Sugishita, J. High Energy Phys. 11 (2017) 203.

[33] R. Choi and A. Akhoury, J. High Energy Phys. 09 (2019) 031.

[34] M. Mirbabayi and M. Porrati, Phys. Rev. Lett. 117, 211301 (2016).

[35] B. Gabai and A. Sever, J. High Energy Phys. 12 (2016) 095.

[36] D. Carney, L. Chaurette, D. Neuenfeld, and G. Semenoff, J. High Energy Phys. 09 (2018) 121. 